\title{
The clinical utilities of multi-pinhole single photon emission computed tomography
}

\author{
Ilker Ozsahin ${ }^{1,2,3}$, Ling Chen $^{1}$, Arda Könik ${ }^{4}$, Michael A. King ${ }^{5}$, Freek J. Beekman ${ }^{6,78}$, Greta S. P. Mok ${ }^{1,9}$ \\ ${ }^{1}$ Biomedical Imaging Laboratory, Department of Electrical and Computer Engineering, Faculty of Science and Technology, University of Macau, \\ Macau, China; ${ }^{2}$ Department of Biomedical Engineering, Faculty of Engineering, Near East University, Nicosia/TRNC, Mersin-10, Turkey; \\ ${ }^{3}$ DESAM Institute, Near East University, Nicosia/TRNC, Mersin-10, Turkey; ${ }^{4}$ Department of Imaging, Dana Farber Cancer Institute, Boston, MA, \\ USA; ${ }^{5}$ Department of Radiology, University of Massachusetts Medical School, Worcester, MA, USA; ${ }^{6}$ Section of Biomedical Imaging, Department \\ of Radiation Science and Technology, Delft University of Technology, Mekelweg 15, 2629 JB Delft, The Netherlands; ${ }^{7}$ MILabs B.V, Heidelberglaan \\ 100, 3584 CX, Utrecht, The Netherlands; ${ }^{8}$ Department of Translational Neuroscience, Brain Center Rudolf Magnus, University Medical Center \\ Utrecht, The Netherlands; ${ }^{9}$ Center for Cognitive and Brain Sciences, Institute of Collaborative Innovation, University of Macau, Macau, China
}

Correspondence to: Greta S. P. Mok. Biomedical Imaging Laboratory, Department of Electrical and Computer Engineering, Faculty of Science and Technology, University of Macau, Macau, China. Email: gretamok@um.edu.mo.

\begin{abstract}
Single photon emission computed tomography (SPECT) is an important imaging modality for various applications in nuclear medicine. The use of multi-pinhole (MPH) collimators can provide superior resolution-sensitivity trade-off when imaging small field-of-view compared to conventional parallel-hole and fan-beam collimators. Besides the very successful application in small animal imaging, there has been a resurgence of the use of MPH collimators for clinical cardiac and brain studies, as well as other small fieldof-view applications. This article reviews the basic principles of $\mathrm{MPH}$ collimators and introduces currently available and proposed clinical MPH SPECT systems.
\end{abstract}

Keywords: Single photon emission computed tomography (SPECT); multi-pinhole; collimator; brain; cardiac

Submitted Jul 08, 2020. Accepted for publication Jul 30, 2020.

doi: 10.21037/qims-19-1036

View this article at: http://dx.doi.org/10.21037/qims-19-1036

\section{Introduction}

The increasing clinical need for dedicated diagnostic systems with improved performance for imaging specific organs, e.g., the heart and brain, has led to the development of single photon emission computed tomography (SPECT) systems with better resolution, sensitivity and throughput (1-12) than standard general-purpose SPECT systems. Over the years, SPECT has become a powerful tool for clinical imaging, e.g., myocardial perfusion (13-16), Alzheimer's disease (AD) (17-21), Parkinson's disease (PD) (22-25), thyroid disease $(26,27)$, cancer $(28)$ and theranostic applications (29).

SPECT is based on the detection of single gamma rays emitted from an administered radiotracer that is distributed in the patient's body. The detected gamma rays are transformed into electronic signals to form $2 \mathrm{D}$ projection images from which the $3 \mathrm{D}$ map of the radiotracer distribution in the body is reconstructed. During this process, determination of the incident angle of gamma-photons on the detector is crucial. This is done through the usage of a collimator which acts as the "lens" for the gamma-ray detector. A collimator is made of high-atomic number and high-density materials such as tungsten and lead which highly absorb incident photons except those traveling through holes of known angulation. In this way the incident angle of the detected gammarays is determined. The design of the collimator, e.g., hole size, material, overall geometry, etc, determines the spatial resolution and sensitivity of a SPECT system. Spatial resolution is often expressed as full-width-at-half-maximum 
(FWHM) of the spatially variant point spread function, which is related to the intrinsic resolution of the detector and the collimator geometry. On the other hand, sensitivity, which is also spatially variant, is the ratio of the detected photons to the emitted photons and can be expressed as detected percentage or as cps/MBq (SI unit of radioactivity). Collimators with larger holes allow more photons to reach the detector, but increasing the hole diameter decreases the spatial resolution since the collimator is less selective with regard to where the photons come from. Conversely, collimators with smaller holes provide better spatial resolution with lower sensitivity. This trade-off needs to be considered for different applications and gamma-ray energy to determine the optimal collimator design for a certain application. A comprehensive review of SPECT collimator selection, optimization, and fabrication can be found in (30).

For imaging smaller objects than the detector (e.g., organs or tumors), the trade-off between spatial resolution and sensitivity compared to a conventional parallel-hole collimator can be improved upon with the use of converging collimators for organ-specific SPECT applications (31). For example, the heart or the brain can be imaged much more efficiently by fan beam, cone beam or focused pinhole collimators, which can result in better trade-off in spatial resolution and sensitivity (32). Multi-pinhole (MPH) collimators have been proposed to address resolutionsensitivity tradeoff issues together with sampling issues (33). Besides the increased sensitivity from a greater number of pinholes when the pinholes are focused to cover a common volume, MPH collimators can obtain multiple projections at a single detector position, which can improve the angular and axial sampling compared to the single pinhole collimator (34-36). The increased sensitivity and sampling allow for improved image quality, shorter acquisition time and/or lower injection dose. Another advantage of MPH collimators is the large reduction in collimator penetration versus parallel-, cone- or fan-beam collimators. This is particularly an issue for the case of down-scatter from higher-energy photons, as for those emitted by ${ }^{123} \mathrm{I}$ with $\sim 2-3 \%$ abundance in addition to its primary photon. Könik et al. demonstrated that for ${ }^{123} \mathrm{I}$ DaTscan imaging, total scatter, multiple scatters, and penetration events for the low energy high resolution (LEHR) parallel-hole collimators were 2.5, 7.6 and 14 times more than that of an MPH collimator within the $15 \%$ photopeak window and with a 2-cm thick tungsten aperture plate (37).

In 2007 Beekman and van der Have (1) provided a comprehensive review on the development of pinhole collimated SPECT technology for preclinical and clinical applications. Since then MPH collimation was investigated and applied more and more often for clinical applications. Yet the purpose of this review is to present an overview of clinical MPH SPECT systems that have been proposed, some of which are clinically available. Technical considerations such as pinhole aperture design, effect of multiplexing, sampling and depth of interaction (DOI) are firstly presented in the next section. Various dedicated clinical cardiac, brain, thyroid, and breast MPH SPECT systems are then presented in the following sections.

\section{Technical considerations for MPH collimators}

\section{Pinhole aperture}

The most commonly used materials for clinical pinhole SPECT collimator are tungsten $(\mathrm{W})$ and lead $(\mathrm{Pb})$ due to their high stopping power and economic considerations. Smith et al. (38) investigated $\mathrm{W}$ and $\mathrm{Pb}$ as the pinhole material for ${ }^{131} \mathrm{I}$ intratumor imaging. Imaging ${ }^{131} \mathrm{I}$ tracers is challenging because of the high energy photons (primary emission at $364 \mathrm{keV}$ and secondary emissions at 284, 637, and $723 \mathrm{keV}$ ), which cause more penetration near the pinhole aperture. They suggested that $W$ has advantages over $\mathrm{Pb}$ in this application, likely due to the fact that $\mathrm{W}$ has a higher linear attenuation coefficient than $\mathrm{Pb}$, i.e., $4.10 \mathrm{~cm}^{-1}$ vs. $2.97 \mathrm{~cm}^{-1}$ at $364 \mathrm{keV}$ (39). Tornai et al. (40) compared $W$ and depleted uranium-238 (DU-238) pinhole apertures with different pinhole diameters $(1,2,3$, and $4 \mathrm{~mm}$ ) for imaging breast lesions using ${ }^{99 \mathrm{~m}} \mathrm{Tc}$ tracers. The performance of $\mathrm{W}$ and DU-238 was found to be similar, while pinholes with $2-3 \mathrm{~mm}$ aperture sizes had the best results in terms of signal-to-noise ratio (SNR). Gold, uranium and platinum are other possible clinical pinhole materials that require further investigation, and Nguyen et al. showed that gold can be beneficial for high energy isotopes such as ${ }^{111} \operatorname{In}(41)$.

Bal et al. (42) suggested that there are three possible geometries for the pinhole, i.e., non-focusing right-circular double-cone (NFRCDC), focusing right-circular doublecone (FRCDC) and focusing oblique-circular double-cone (FOCDC), as shown in Figure 1. The choice of aperture depends on the photon energy for the specific application. For instance, FOCDC is shift-invariant for low and medium energy, which is good for reducing reconstruction time. However, it is only suitable for a small FOV and difficult 


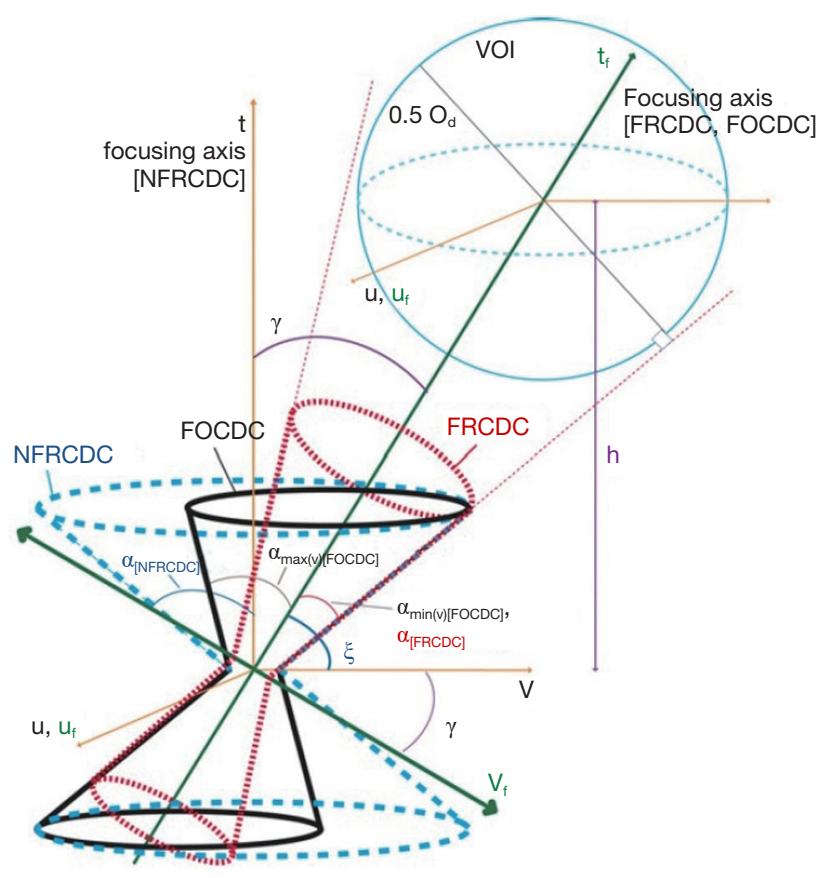

Figure 1 Schematic sketch of NFRCDC, FOCDC, and FRCDC knife-edge pinhole designs [courtesy of Bal et al. (42)].

to fabricate. FRCDC has the least penetration owing to its smallest acceptance angle under the same FOV, making it the best for high energy applications.

The sensitivity $(S)$ of a knife-edge single pinhole collimator with a normal axis can be described by Eq. [1] (43).

$$
\begin{aligned}
S_{\text {knife }}(\theta, l)= & \frac{d^{2} \sin ^{3} \theta}{16 l^{2}}+\frac{\sin ^{5} \theta \tan ^{2} \frac{\alpha}{2}}{8 l^{2} \mu^{2}} \\
& \sqrt{1-\frac{\cot ^{2} \theta}{\tan ^{2} \frac{\alpha}{2}}}\left(1-\frac{\cot ^{2} \theta}{\tan ^{2} \frac{\alpha}{2}}+\mu d \csc \theta \cot \frac{\alpha}{2}\right)
\end{aligned}
$$

where $d$ is the physical hole diameter, $\theta$ is the incident angle, $l$ is the imaging distance, $\mu$ is the attenuation coefficient of the aperture material, and $\alpha$ is the acceptance angle of the pinhole (Figure 2).

The total sensitivity of an MPH collimator can be written as a summation over the sensitivities of the individual pinholes as in Eq. [2].

$$
S_{n}=\sum_{i=1}^{n} S_{i}
$$

where $n$ is the number of pinholes. The system spatial resolution of a single pinhole collimator can be described

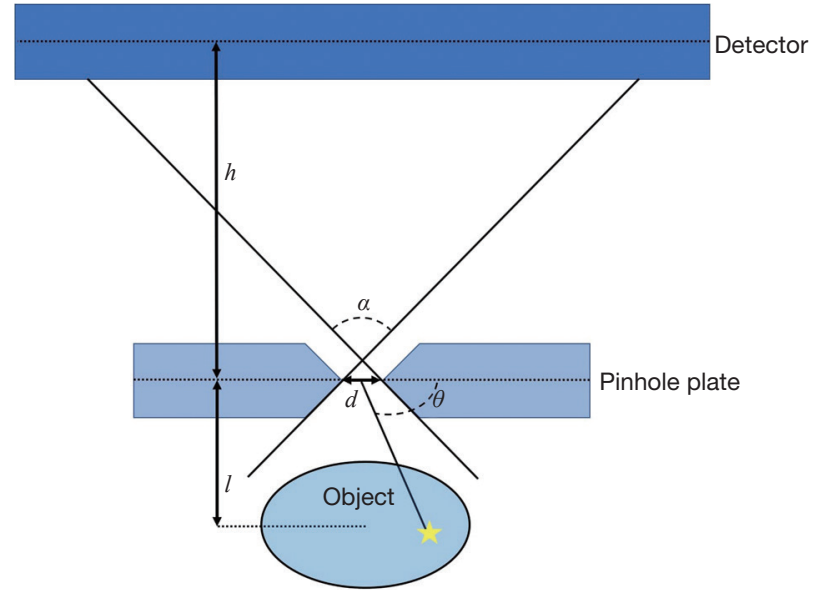

Figure 2 Pinhole imaging parameters. Dashed lines inside the detector and the pinhole plate show their central plane, respectively. The yellow star represents the origin of the gamma ray.

by Eq. [3].

$$
R_{s}=\sqrt{\left(\frac{l}{h} R_{i}\right)^{2}+\left(R_{g}\right)^{2}}=\sqrt{\left(\frac{l}{h} R_{i}\right)^{2}+\left(d_{\text {Reff }} \times \frac{l+h}{h}\right)^{2}}
$$

where $R_{s}$ is the system resolution, $b$ is the aperture-todetector distance (Figure 2), $R_{i}$ is the intrinsic resolution of the detector, $R_{g}$ is geometric resolution, and $d_{\text {Reff }}$ is the effective pinhole diameter corrected for penetration effect, which is given by Eq. [4].

$$
d_{\text {Reff }}=d+\frac{\ln 2}{\mu} \tan \frac{\alpha}{2}
$$

Besides the knife-edge pinhole, the keel-edge (also known as channeled-edge) pinhole is proposed by Smith et al. $(38,44)$ for penetration reduction in pinhole collimation, especially in high resolution imaging using high-energy gamma photons. The shape of keel-edge collimators minimizes gamma penetration near the edge of the aperture (Figure 3). They found that increasing the channel height resulted in a higher spatial resolution but, also a rapid decrease in sensitivity for off-axis sources. Other methods of reducing penetration are based on clustered pinholes $(45,46)$ or the use of smaller pinhole aperture sizes $(43,44)$.

Developing an accurate mathematical model of the sensitivity and resolution profile of the keel-edge collimator is challenging. Wang (47) modelled the sensitivity of a keeledge pinhole collimator as in Eq. [5]. 


$$
S_{\text {keel }}(\theta, l)=\frac{d^{2} \cos ^{3} \theta}{16 l^{2}}\left(1-\frac{\eta}{\pi}-\frac{\sin (\eta)}{\pi}\right)
$$

where $\eta$ is defined as in Eq. [6].

$$
\sin \frac{\eta}{2}=k \tan |\theta| / d \Rightarrow \eta=2 \sin ^{-1}(k \tan |\theta| / d)
$$

where $k$ is the channel height.

In recent designs, apertures in $\mathrm{MPH}$ collimators are usually tilted towards an external focal point to focus on the center of FOV (CFOV) and increase the sensitivity. However, modelling the sensitivity and resolution of the tilted pinholes is challenging and conventional formulas cannot be applied because of the position-dependent penetration length and DOI of the tilted aperture (as detailed in later section). Huang et al. (48) derived an analytical sensitivity calculation method for tilted knifeedge apertures based on a modification of the conventional formula for normal knife-edge apertures. To correct this geometric change, a virtual object plane parallel to the tilted aperture plane was set such that the geometric sensitivity and penetration path length in the conventional equation can be applied with respect to this virtual object plane. The sensitivity for the tilted pinholes can be given as follows:

$$
\begin{aligned}
& S_{\text {tilt }}\left(\theta^{\prime}, l^{\prime}\right)=\frac{d_{\text {Seff }}^{2}}{16 l^{\prime 2}} \sin ^{3} \theta^{\prime} \\
& d_{\text {Seff }}=\sqrt{d\left(d+\frac{2}{\mu} \tan \frac{\alpha}{2}\right)+\frac{2}{\mu^{2}} \tan ^{2} \frac{\alpha}{2}} \\
& l^{\prime}=\frac{\left|n_{x} x_{1}+n_{y} y_{1}+n_{z} z_{1}-n_{x} i-n_{y} j-n_{z} k\right|}{\sqrt{n_{x}^{2}+n_{y}^{2}+n_{z}^{2}}} \\
& \theta^{\prime}=\frac{\pi}{2}-\cos ^{-1} \\
& \left(\begin{array}{l}
n_{x}\left(x_{1}-i\right)+n_{y}\left(y_{1}-j\right)+n_{z}\left(z_{1}-k\right) \\
\left(\sqrt{n_{x}^{2}+n_{y}^{2}+n_{z}^{2}}\right)\left(\sqrt{\left(x_{1}-i\right)^{2}+\left(y_{1}-j\right)^{2}+\left(z_{1}-k\right)^{2}}\right)
\end{array}\right) \\
& \vec{n}=\left(x_{2}-x_{1}, y_{2}-y_{1}, z_{2}-z_{1}\right)=\left(n_{x}+n_{y}+n_{z}\right)
\end{aligned}
$$

where $d_{\text {Seff }}$ is the sensitivity effective pinhole diameter corrected for penetration effect (49), $\vec{n}$ is a normal vector coincident with the tilted pinhole axis, $\left(\mathrm{x}_{1}, \mathrm{y}_{1}, \mathrm{z}_{1}\right)$ is the pinhole aperture center and $\left(\mathrm{x}_{2}, \mathrm{y}_{2}, \mathrm{z}_{2}\right)$ is an arbitrary point along the pinhole axis. To the best of our knowledge, there
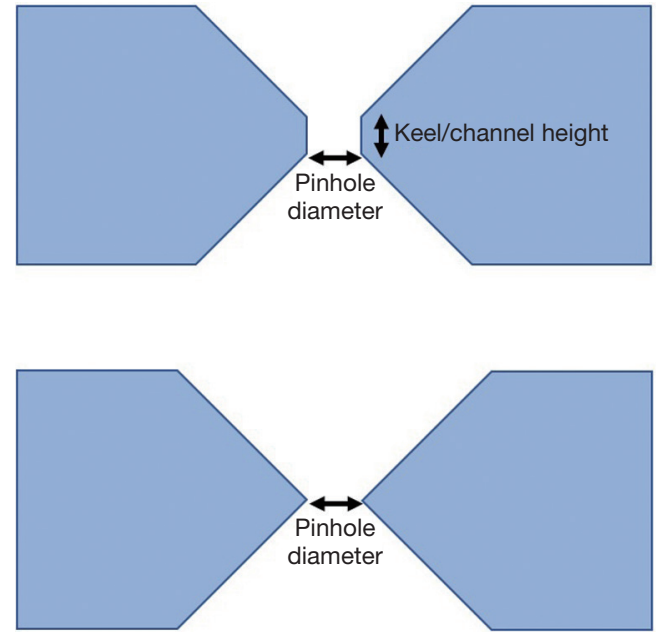

Figure 3 Schematic sketch of keel-edge (top) and knife-edge (bottom) pinhole designs.

has been no study published in the literature on the analytic modeling of the resolution of a titled pinhole for $\mathrm{MPH}$ collimators.

U-SPECT systems $(50,51)$ employ dual stage collimation to effectively achieve non-multiplexing, i.e., non-overlapping projections (Figure 4). In these systems, 75 gold circular pinholes were arranged in a cylindricalshaped collimator and there is another outer lead cylinder with square holes that act as a shielding for the detector, which completely solves the problem of multiplexing as discussed in the next section.

An adaptation to this method that also allows a circular pinhole aperture but provides rectangular projections to avoid multiplexing is the use of a lofthole proposed by Deprez et al. (52). Another aperture design that uses squareor rectangular-pinholes with a square or rectangular FOV was proposed by Metzler et al. (53) and further developed by Xia et al. (54). Rectangular pinholes consist of doublepyramid-shaped openings with a square or rectangular intersection of two pyramids (Figure 5) (55). The main advantage of the rectangular FOV compared to a circular FOV is that the projections can be packed more efficiently.

\section{Multiplexing effects}

Several strategies have been attempted to further improve the detection efficiency in MPH SPECT (30). One of them is to allow overlapping of projections, i.e., multiplexing, from different pinholes (56). The degree of multiplexing 
A

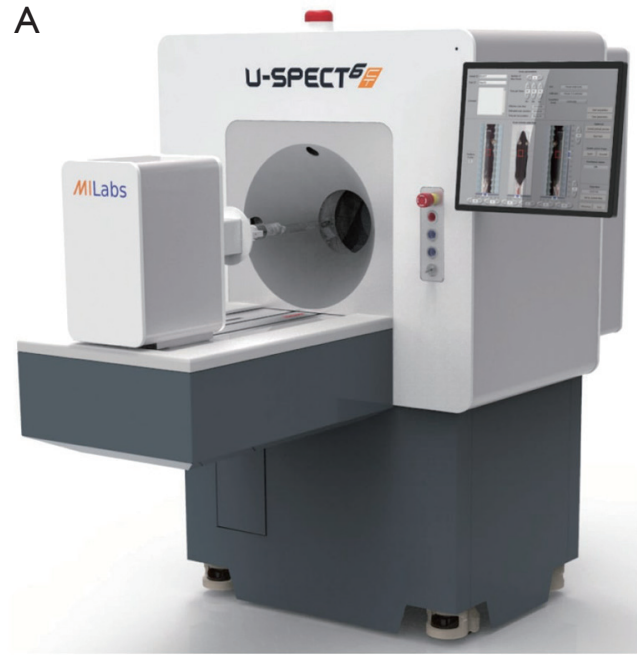

B

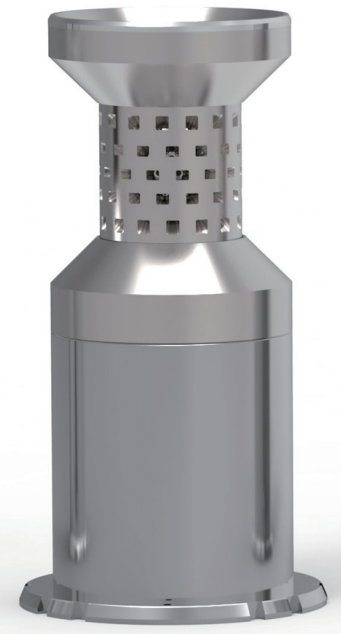

Figure 4 Dual stage collimation with circular pinholes and an outer lead shield with square holes. (A) A U-SPECT system and (B) its corresponding dual stage MPH collimator. With permission of MILabs B.V. Utrecht, The Netherlands.
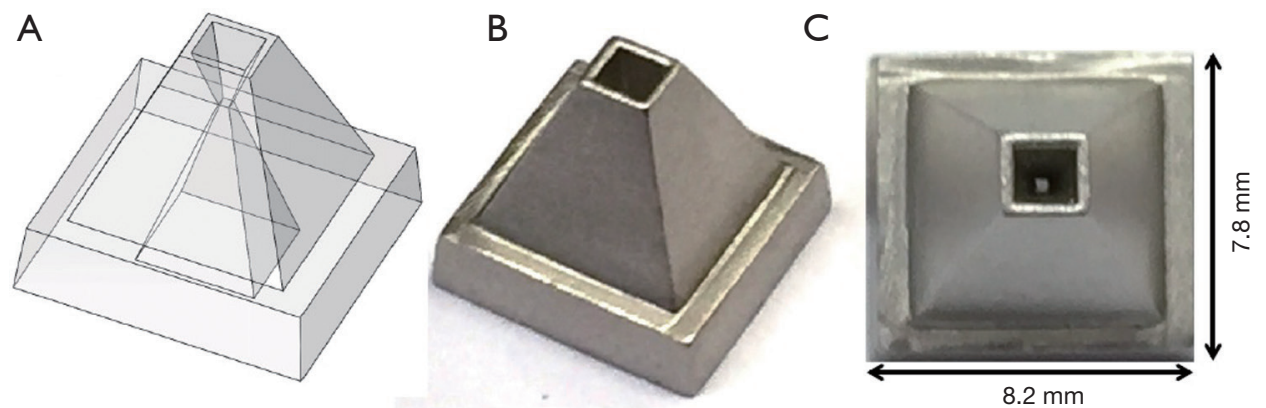

Figure 5 Rectangular pinholes with rectangular FOV. (A) Semitransparent design drawing of a rectangular pinhole insert, showing the double-pyramid-shaped inside walls, which open up above and below the plane of the small square aperture. (B) Photograph of one of the many identical platinum-iridium inserts. (C) Top view showing the $0.3-\mathrm{mm}$ square-pinhole aperture, located $2 \mathrm{~mm}$ below the opening on the top, and $5 \mathrm{~mm}$ above the large opening on the bottom [courtesy of Moore et al. (55)].

can be defined as Eq. [12] (57).

$$
\text { Multiplexing } \%=\frac{\mathrm{P}_{\text {multiplexing }}}{\mathrm{P}_{\text {non-zero }}}
$$

where $\mathrm{P}_{\text {multiplexing }}$ and $\mathrm{P}_{\text {non-zero }}$ represent the number of pixels with multiplexing in the projection and number of pixels with non-zero value in the projection, respectively. Since multiplexing allows the projection size to be larger than that of the non-multiplexing design, it could potentially allow a larger magnification factor to improve spatial resolution and increase the sensitivity if the larger magnification is obtained by bringing the pinholes closer to the source. On the other hand, without changing magnification, more pinholes could be "packed" to further improve the detection efficiency at the same spatial resolution. However, the increased detection efficiency from multiplexing does not guarantee an improvement in the SNR. The projections with multiplexing might lead to artifacts in image reconstruction because of the overlapped ambiguous region where detected events could have come through more than one aperture. Several research groups have shown that multiplexing may misplace the activity in the reconstruction (56-59). Van Audenhaege et al. suggested that more multiplexing does not necessarily result in more artifacts as long as the non-multiplexed data is complete (60). The effects of multiplexing are associated with the activity 


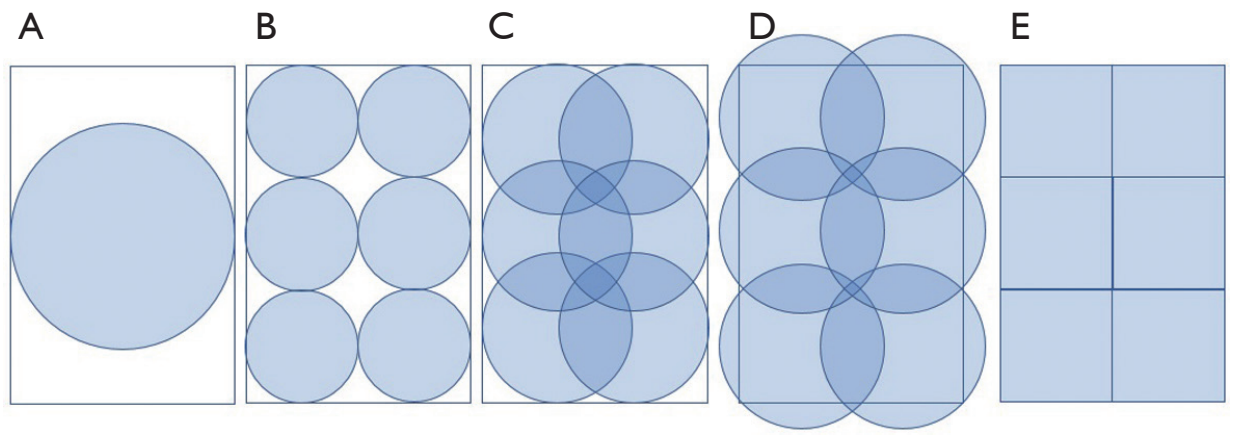

Figure 6 Simplified illustration of projection images generated using a spherical phantom. The ratio of utilized area to total area of detector is $\sim 52 \%$ and $\sim 79 \%$ for (A) single pinhole and (B) six-pinhole collimators with no multiplexing, respectively. For (C), there is $~ 93 \%$ usage with $\sim 33 \%$ multiplexing and no truncation. In (D), there is $100 \%$ usage with $~ 38 \%$ multiplexing and truncation. For (E), lofthole, dual stage collimation and rectangular pinhole, there is $100 \%$ usage with no multiplexing, while truncation may or may not be present depending on the magnification of the spherical phantom.

distribution (54) and sampling, i.e., the number of pinholes, their pattern and truncation.

The multiplexing effect can be reduced by several strategies, including baffle (61), the aforementioned dual stage collimation, loftholes, and rectangular pinholes. The adverse effect of multiplexing can also be corrected during iterative image reconstruction (62). This algorithm estimates both the values of all image voxels, and the demultiplexed projection data from each of the pinholes. Thus, the reconstruction procedure alternates between two different iterative processes, i.e., first, estimating separated projection data for each pinhole and second, using the separated projection estimates to reconstruct an image. These two iterative steps are repeated until the multiplexing artifacts are considered to be eliminated or adequately reduced.

Figure 6 illustrates the MPH multiplexing with a rectangular detector. It compares the projections of a spherical phantom with one-pinhole and four different cases of six-pinhole collimator with varying degrees of multiplexing, truncation, and utilization of detectors.

\section{Pinbole pattern and sampling}

MPH collimators not only can provide superior detection efficiency, they can also improve image quality by offering multiple axial and angular projections in a single acquisition view. For single pinhole imaging, only the central plane of the object has complete sampling. Distortions manifest outside this region, especially towards the edge of the object in the axial direction (Figure 7). Vanhove et al. showed that the axial spatial resolution and sensitivity of single pinhole SPECT system can be improved by using threepinhole collimators (35). DiFilippo et al. (36) showed that the artifacts reduced when either helical sampling (bed movement in constant intervals between angular steps) or order-subsets sampling (bed movement in a repeating sequence to different axial positions) were utilized, since both methods provide improved axial sampling. Vaissier et al. (63) developed spiral trajectories of the animal bed to improve temporal resolution and reduce image reconstruction time. This technique requires fewer acquisition steps compared to other stationary small animal SPECT systems with focusing MPH collimators, which need to shift the animal bed for 5 to 80 bed positions for a total body mouse scan depending on the collimator used. For the cases where many bed positions are needed, the acquisition time could be reduced dramatically by helical trajectories.

Mok et al. (37) found that the MPH SPECT for small FOV imaging is able to achieve high detection efficiency without much deterioration in resolution, as MPH provided more angular sampling compared to conventional LEHR and single pinhole collimator. Funk et al. (64) proved that MPH collimators with nine pinholes reduced the axial artifacts and distortion compared to single pinhole for the same number of angular views. As shown in Figure 8, a low number of pinholes in a regular arrangement combined with multiplexing may result in "ghost" artifacts, i.e., for 4-pinhole in this case. The degree of multiplexing is more in this 5-pinhole case, yet the "ghost" artifacts are not observed, probably due to the added central projection 


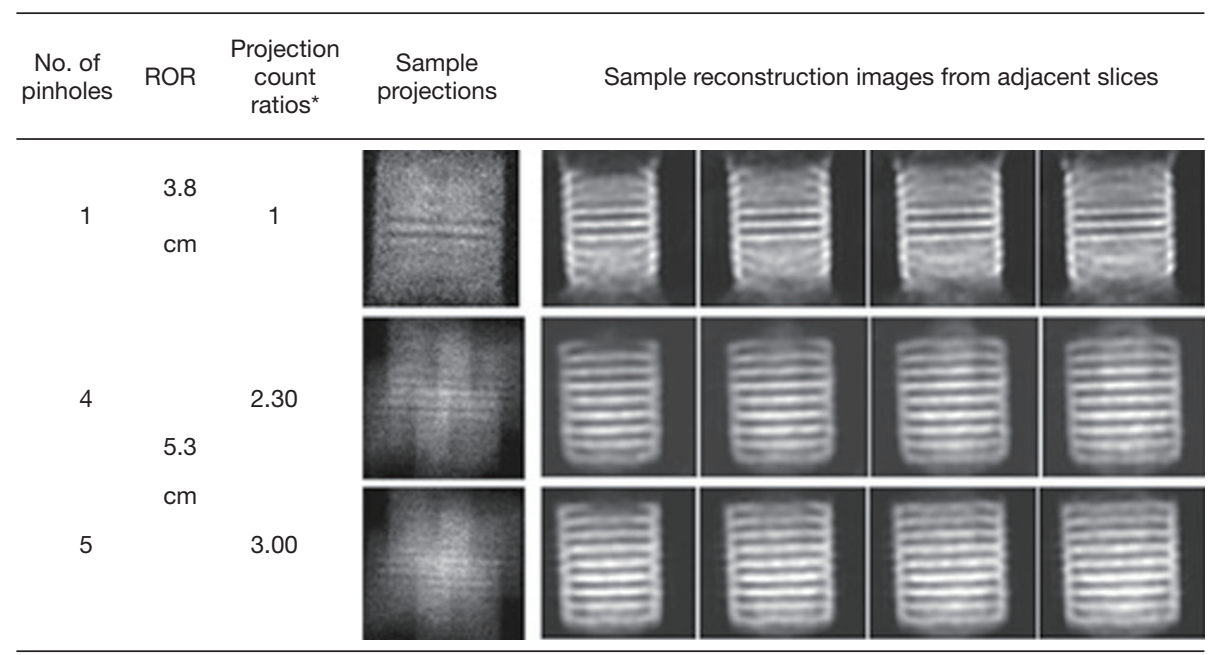

*with same acquisition time

Figure 7 Experimental projections and axial SPECT images from the Defrise phantom imaged under different pinhole configurations [courtesy of Mok et al. (37)].

providing more information for image reconstruction to avoid the null space problem. Thus, pinholes should be arranged carefully to avoid artifacts, and maximize axial and angular sampling to improve the reconstructed image quality (65).

\section{DOI}

When gamma rays interact with the detector, the exact location of interaction through the detector along the depth direction is usually unknown. This effect causes blurring in the final reconstructed image and is called the DOI or parallax effect. The DOI effect can be negligible for gamma rays interacting with the detector at a right angle. However, when gamma rays pass through the pinhole and interact with the detector at an oblique angle, particularly for photons detected towards the edge of the detector, the photon interaction paths would be more "spread out", causing errors in the recorded position and deteriorating spatial resolution (Figure 9). Projections from the pinhole collimator may suffer more from the DOI effect compared to other collimators when the incident angles are large. Hwang et al. (66) performed simulation studies to quantify the spatial resolution loss due to the DOI effect for $140 \mathrm{keV}$ gamma rays. The simulations were performed with CsI and high purity Germanium detectors with thicknesses of 6 and $10 \mathrm{~mm}$, respectively. They estimated that the inplane (normal to the axis of rotation) resolution loss was
0.32-0.63 mm FWHM and the cross-plane (aligned with the axis of rotation) resolution loss was $0.21-0.42 \mathrm{~mm}$ FWHM. Some research groups are working on the elimination of the DOI effect for MPH SPECT. Beekman et al. (67) designed a high-resolution detector that directs CsI fibers towards the pinhole so that the scintillation light can be transported along the same line as the gamma ray travels before it interacts with the crystal. Furthermore, Korevaar et al. (68) employed a curved shape scintillation crystal and a dedicated optic fiber bundle to eliminate the spatial resolution loss from DOI effectively.

\section{Quantitative MPH SPECT}

Advanced image reconstruction algorithms such as statistical reconstruction methods that incorporate corrections for various image degradation factors, e.g., attenuation, object scatter, patient motion, partial volume effect and collimatordetector response functions that include geometric response function, intrinsic response function, septal penetration, septal scatter function, as well as multiplexing and the DOI effect mentioned in the previous sections, are essential for pursuing absolute quantitation in MPH SPECT (69). These corrections are modeled within the iterative reconstruction scheme. Compared to simple analytical reconstruction methods such as filtered back-projection, statistical reconstruction methods can incorporate the random nature of photon detection and allow compensation 


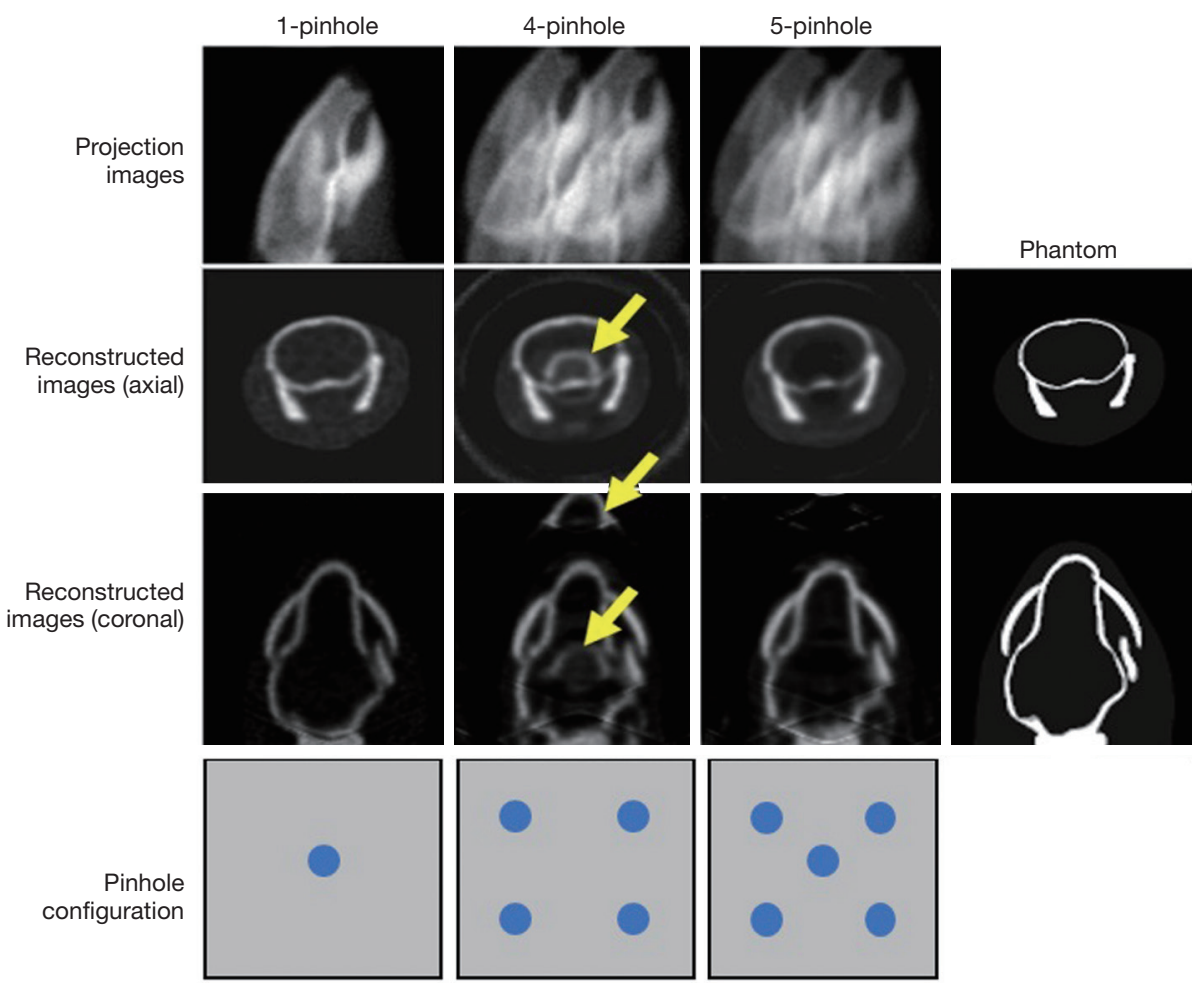

Figure 8 Monte Carlo simulations of a digital mouse phantom with different numbers of pinholes. Significant artifacts occur in the regular 4-pinhole pattern with multiplexing (yellow arrows) while they are not seen as significantly in the 5-pinhole pattern with a higher degree of multiplexing.

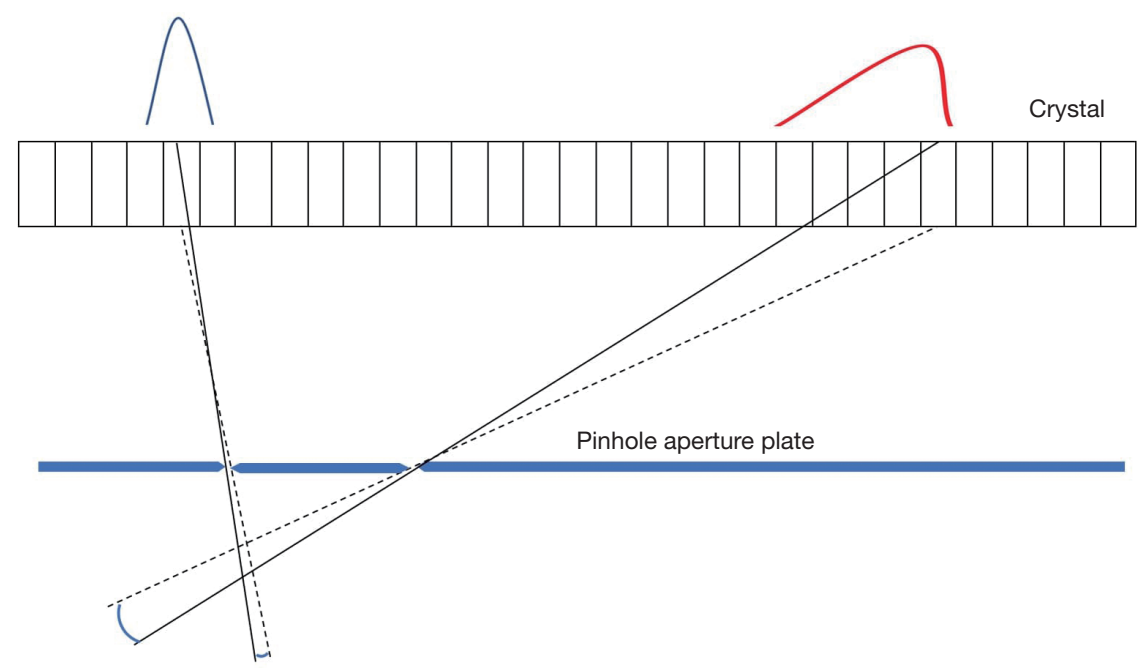

Figure 9 Parallax or DOI effect due to gamma-rays entering the detector at oblique angles. Photons being detected with a larger incident angle would be more blurred out (red point spread function) compared to those with a smaller incident angle (blue point spread function).

of various image quality degradation effects in the acquisition process via accurate system matrix modelling (70). The reconstructed image quality is significantly improved by the statistical reconstruction method but at the cost of increased computational burden. The computation time of statistical reconstruction for MPH is longer compared to the single 

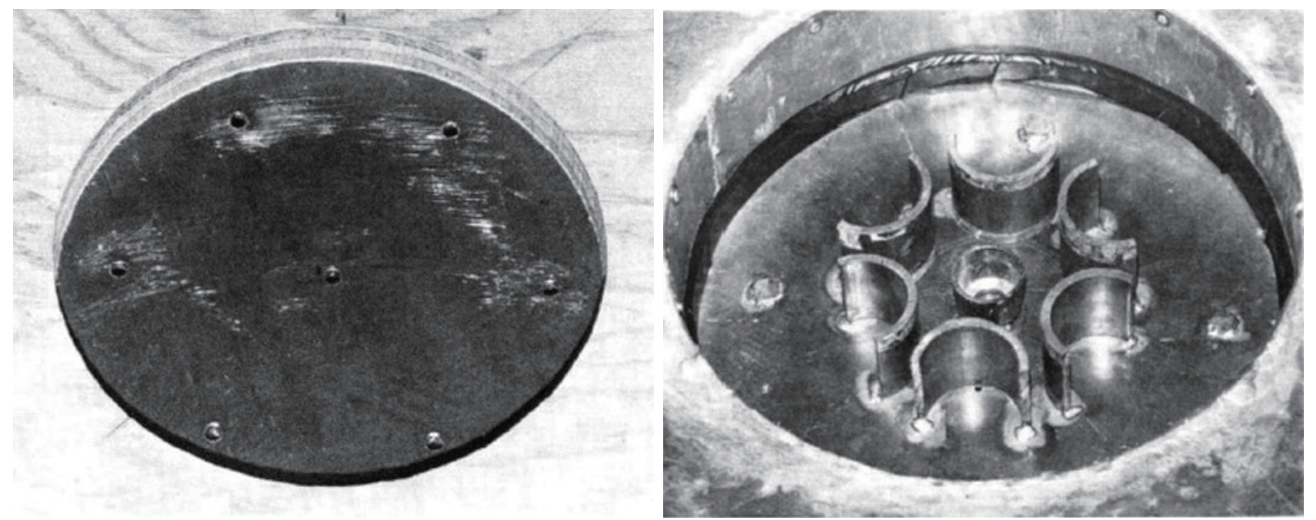

Figure 10 Seven-pinhole array. Left: close-up view of the collimator at the entrance. Right: the exit surfaces that has shielding to prevent projection overlapping [courtesy of Vogel et al. (33)].

pinhole and increases with the pinhole number. For MPH SPECT reconstruction, the system response matrix can be calculated based on Monte Carlo simulation or analytical techniques, and can be combined with an experimental characterization (71-73). However, obtaining an experimental and Monte Carlo based system response matrix is time consuming even when interpolations and variance reduction methods (74,75), e.g., force detection techniques, are applied.

\section{Clinical MPH SPECT systems}

\section{7-pinhole collimator with Anger camera}

One of the first MPH collimators was developed in 1978 when Vogel et al. (33) used seven pinholes to obtain simultaneous projections of an emission source onto seven independent regions of an Anger scintillation camera. The lead collimator of this MPH system, shown in Figure 10, had pinhole aperture diameters of $5.5 \mathrm{~mm}$. The six peripheral pinholes are in a hexagonal pattern at a distance of $6.35 \mathrm{~cm}$ from the central pinhole, which is positioned at the center of the hexagon. Lead shielding was used inside the collimator to prevent multiplexing of the pinhole projections. The acceptance angle of the central pinhole is $53^{\circ}$ and perpendicular to the crystal surface, while the acceptance angle of the outer holes is $45^{\circ}$ and their longitudinal axes converge inward at $26.5^{\circ}$, forming a conically tipped cylindrical FOV $12.7 \mathrm{~cm}$ in diameter. Spatial resolution was found to be $1.0 \mathrm{~cm}$ at $12.7 \mathrm{~cm}$ from the pinhole plate and the system sensitivity was found to be $750 \mathrm{cps} / \mathrm{MBq}$ at $11.5 \mathrm{~cm}$ from the pinhole plate (76).

\section{FASTSPECT}

FASTSPECT is a high-resolution dynamic MPH SPECT system dedicated to clinical brain imaging originally proposed by Rowe et al. in 1993 (77) and developed by Klein et al. in 1995 (78). The originally proposed system consists of 20 stationary modules, 100 pinhole aperture of $1.4 \mathrm{~mm}$ square in a lead-alloy collimator $15 \mathrm{~cm}$ from the focal point, and 20 modular camera oriented tangent to the hemisphere surface at $22 \mathrm{~cm}$ from the focal point. The system was able to collect $3 \mathrm{D}$ projections in $50 \mathrm{~ms}$ to generate dynamic $3 \mathrm{D}$ images at 20 frames per second. At the focal point of the apertures the spatial resolution and sensitivity of the system are $4.8 \mathrm{~mm}$ and $973.0 \mathrm{cps} / \mathrm{MBq}$, respectively.

\section{MPH myocardial perfusion imaging (MPI) SPECT}

Funk et al. (64) proposed an MPH SPECT system for MPI to increase the sensitivity by 5 -fold while maintaining the same image quality compared to a conventional rotational SPECT system with a low-energy general-purpose (LEGP) parallel hole collimator. They mounted a 9-pinhole collimator to a standard gamma camera (Philips Argus) and nine simultaneous projections of the myocardium were projected onto the $50 \mathrm{~cm} \times 38 \mathrm{~cm}$ active area of the detector with no multiplexing. Pinholes with a diameter of $8 \mathrm{~mm}$ were arranged on a $30 \mathrm{~cm} \times 20 \mathrm{~cm}$ plate in a rectangular array so that the focal point was $31.5 \mathrm{~cm}$ away from the detector surface (Figure 11). Aperture-to-detector distance was set to $12 \mathrm{~cm}$. The spatial resolution of the point source was measured as $13 \mathrm{~mm}$ (vs. $10 \mathrm{~mm}$ for LEGP) at $10 \mathrm{~cm}$ 

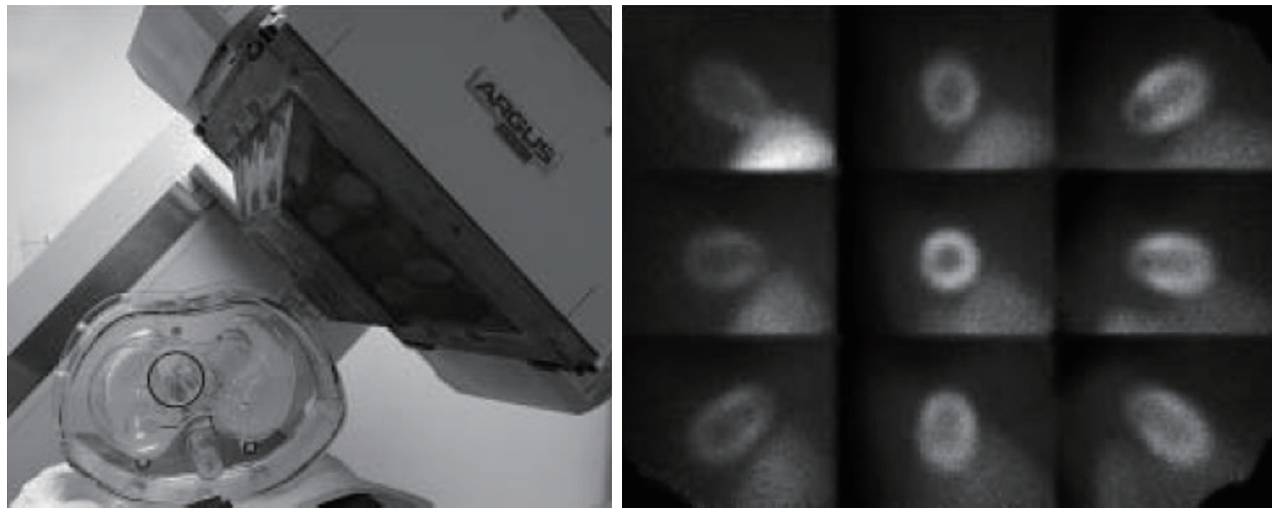

Figure 11 Left: experimental setup of the 9-pinhole MPI SPECT system with a torso phantom. Right: non-multiplexed projection data of the phantom with cardiac and liver uptakes [courtesy of Funk et al. (64)].
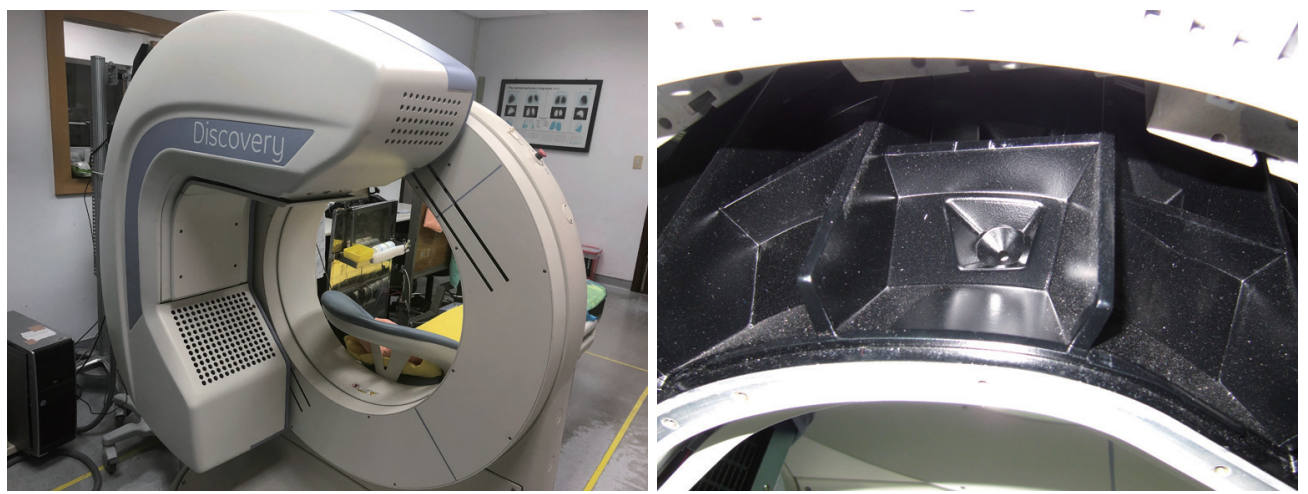

Figure 12 Left: Discovery NM 530c featuring a gantry that is similar to a conventional cardiac SPECT camera, with a different detector assembly that allows completely stationary acquisition. Right: sample pinhole aperture from Discovery NM 530c (courtesy of Taipei General Veterans Hospital).

from the collimator and the sensitivity was 10 -fold higher than that of LEGP at $10 \mathrm{~cm}$ from the collimator.

\section{Discovery NM 530c}

The Discovery NM 530c is a commercially available system dedicated for MPI, developed by GE Healthcare, Haifa, Israel (4). It has a C-shaped gantry, which accommodates multiple cadmium zinc telluride (CZT) detectors (Figure 12). The scanner consists of 19 stationary, $16 \times 16$ pixelated CZT modules of $5 \mathrm{~mm}$ thickness, equipped with pinhole collimators. Each pinhole has an aperture of $5.1 \mathrm{~mm}$ in diameter. The pixel size of the CZT detectors is $2.46 \mathrm{~mm}$. The detectors are arranged in three rows perpendicular to the axial direction. Nine detectors are placed along the transaxial direction covering $180^{\circ}$. Two groups of five detectors are tilted and positioned on each side of the nine detectors in the main row. The intrinsic spatial resolution is the same as the detector pixel size of $2.46 \mathrm{~mm}$, while the system resolution is $6.1 \mathrm{~mm}$ at $10 \mathrm{~cm}$ from the collimator. The overall system sensitivity is $640 \mathrm{cps} / \mathrm{MBq}$ as compared to $400 \mathrm{cps} / \mathrm{MBq}$ of a D-SPECT cardiac system with parallel-holes (79).

\section{Multi-lofthole brain SPECT}

Van Audenhaege et al. (80) designed and simulated a full-ring multi-lofthole brain SPECT based on the modification of an existing PET detector ring made of $24 \mathrm{LaBr}_{3}$ sub-detectors (LaPET, Figure 13). A full-ring multi-lofthole collimator, which consists of a tungsten ring with loftholes, is mounted on the system with a shutter mechanism that controls the 

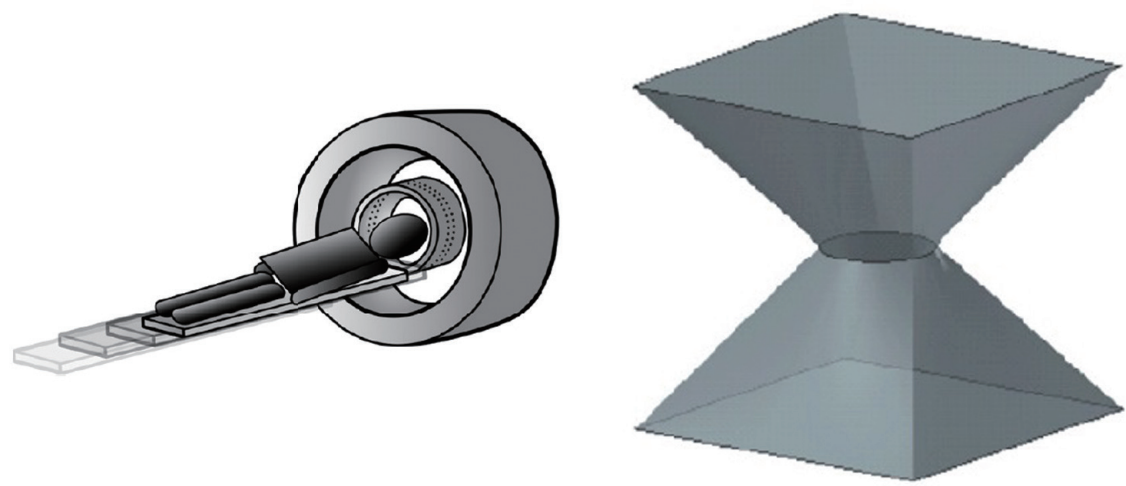

Figure 13 Left: LaPET scanner with a collimator insert. Right: Lofthole with a circular aperture and a rectangular exit window [courtesy of Van Audenhaege et al. (81)].

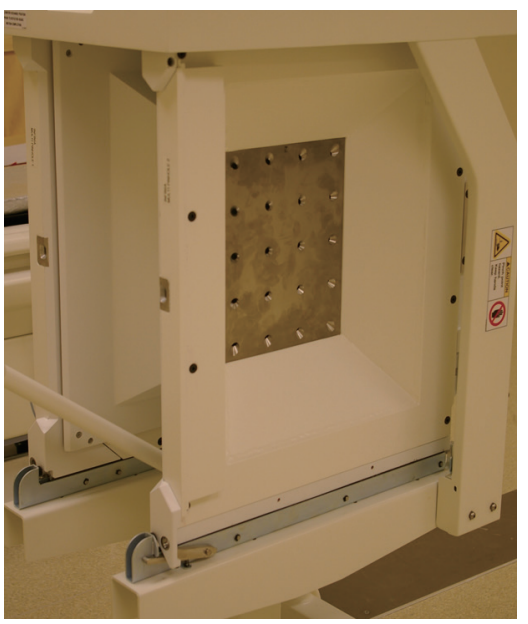

Figure 14 The UCSF 20-pinhole SPECT collimator (courtesy of Dr. Youngho Seo).

projection overlapping and also provides complete angular sampling without the need for rotational acquisition. The researchers fixed the target spatial resolution at $6 \mathrm{~mm}$ in the CFOV and then maximized the volume sensitivity by varying the collimator radius, aperture size and number of loftholes. Their optimization results showed that the volumetric sensitivity $(0.016 \%)$ was 2.5 times lower than the sensitivity of a dual-head system with LEHR collimator, while the spatial resolution was improved, with the ability to reconstruct $4 \mathrm{~mm}$ rods. This stationary system has the potential to be integrated with an MRI system.

\section{0-pinbole SPECT}

The 20-pinhole SPECT collimator (Figure 14) was developed at the University of California, San Francisco and is mounted on a GE Inifinia Hawkeye scanner. It was proposed for MPI (3) striatal dopamine transporter (DaT) imaging (5). The system design parameters are: $19.3 \mathrm{~cm}$ radius of rotation (ROR), $0.75 \mathrm{~cm}$ aperture diameter, $31.5 \mathrm{~cm}$ system radius, $65.4^{\circ}$ acceptance angle and $21 \mathrm{~cm}$ FOV. The pinholes are arranged such that the distances between pinhole centers are 6.2 and $5.8 \mathrm{~cm}$ for column and rows, respectively. Note that all the pinholes are tilted towards the CFOV. Their experimental results showed that the efficiency of this collimator is $1.6 \times 10^{-3}$ which is 22 times of that of LEHR $\left(7.2 \times 10^{-5}\right)$ with 2.8 times resolution loss at $19.3 \mathrm{~cm}$ from the collimator.

\section{DaTscan SPECT system with MPH and fan-beam collimator}

King et al. (7) proposed the placement of an MPH collimator on one head of a SPECT system and a fan-beam collimator on the other head (Figure 15). The MPH collimator provides high resolution and sensitivity for imaging the striatal region and the fan beam collimator provides lower resolution but complete sampling of the brain. The proposed method addresses data sufficiency and a larger volume-of-interest can be covered which includes the occipital lobe for the calculation of striatal binding ratio. The system spatial resolution for the MPH was set to $4.7 \mathrm{~mm}$ at the focal point of the apertures, which is comparable to that of a clinical PET system. At the same location, the sensitivity is $87 \mathrm{cps} / \mathrm{MBq}(0.009 \%)$ which is similar to that of the low energy ultra-high resolution fan beam collimator with an improved resolution $(4.7 v s .7 .4 \mathrm{~mm})$. 

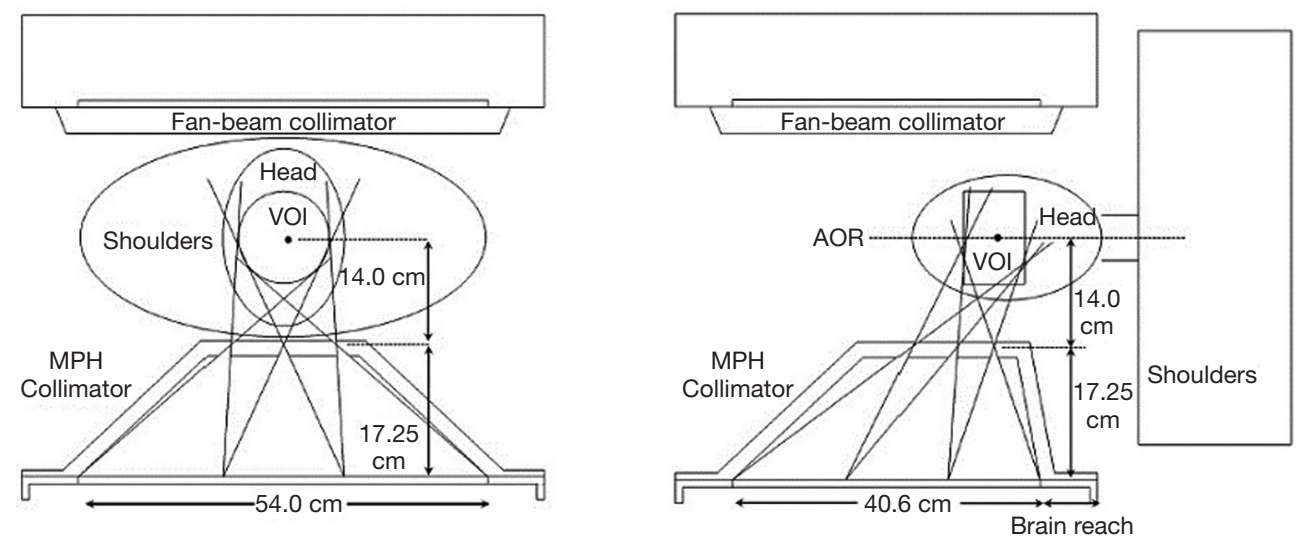

Figure 15 Side views of a combined MPH and fan-beam collimator system [courtesy of King et al. (7)].

\section{G-SPECT-I}

G-SPECT-I $(81,82)$, is a SPECT system developed by TU Delft and MILabs B.V. in Utrecht, Netherlands with interchangeable collimators. The system has a smaller bore collimator for brain, pediatric and large animal imaging (Figure 16) and another collimator for imaging the chest and thorax (e.g., targeted liver, heart and kidney studies) or even a full human body. The design consists of a full ring of stationary detector with intrinsic detector resolution of around $3.5 \mathrm{~mm}$. The "ring" is formed by 9 large FOV cameras using $595 \mathrm{~mm} \times 472 \mathrm{~mm} \mathrm{NaI}(\mathrm{Tl})$ scintillation crystals. The smallest diameter collimator contains 54 pinholes with a bore diameter of $398 \mathrm{~mm}$. A user interface that employs three optical cameras is used to select the scan region to maximize the focus capabilities (83). The smallest rod that can be resolved of a Derenzo resolution phantom with rod dimeter equals to rod distance is $2.5 \mathrm{~mm}$ and $3.5 \mathrm{~mm}$, with a peak sensitivity of $415 \mathrm{cps} / \mathrm{MBq}$ and $896 \mathrm{cps} / \mathrm{MBq}$ (compared to $182 \mathrm{cps} / \mathrm{MBq}$ for a conventional dual-head camera) for the ultrahigh resolution (UHR) collimator and high resolution (HR) collimator respectively. Besides superior spatial resolution, another advantage of G-SPECT-I is its fast scanning speed with sub-second time frames in the case of focused imaging.

\section{9-pinbole brain SPECT}

Chen et al. (10) designed and evaluated two MPH collimators for brain SPECT to image cerebral blood flow and striatum (Figure 17) based on a GE Inifinia Hawkeye scanner. In the standard general-purpose mode (MPGP), the collimator is used for cerebral blood flow imaging, while in the high-resolution mode (MPHR), the collimator is used for DaT imaging. The ROR is set to $167 \mathrm{~mm}$ to provide a FOV of $20 \mathrm{~cm}$ to accommodate the patient's head, without touching the patient's shoulder. Based on theoretical prediction, the MPGP collimator's target resolution is set to $12 \mathrm{~mm}$ and sensitivity is calculated as $0.037 \%$ for the CFOV, while the target resolution and sensitivity of MPHR collimator are $8 \mathrm{~mm}$ and $0.014 \%$, respectively. Both designs have a better performance than the LEHR collimator, which has a resolution of $12 \mathrm{~mm}$ and sensitivity of $0.010 \%$.

\section{Multi-pinbole cardiac SPECT}

Si et al. (8) designed a 14-pinhole cardiac collimator based on a target resolution of $1 \mathrm{~cm}, \mathrm{FOV}$ of $20 \mathrm{~cm}$ and H-mode rotational acquisition for a clinical SPECT scanner by maximizing the sensitivity. The measured FWHM is $8.2 \mathrm{~mm}$, which is $23 \%$ better compared to LEHR with the same imaging distance of $22 \mathrm{~cm}$, while the sensitivity is $0.017 \%$. Yan et al. (9) improved this design for dual-head L-mode stationary and semi-stationary acquisition with a reduced FOV of $16 \mathrm{~cm}$ to further improve the sensitivity and accommodate $95 \%$ of the Chinese population. The aperture size of this 12-pinhole design (Figure 18) is $4.65 \mathrm{~mm}$, while the imaging distance is $18.6 \mathrm{~cm}$ and aperture-to-detector distance is $15.8 \mathrm{~cm}$. Based on the same resolution with the LEHR collimator, the total sensitivity is $0.035 \%$, which is 2.5 times better than LEHR collimator. Semi-stationary acquisition mode with 1-step detector rotation for both detectors showed reduced artifacts compared to the fully stationary acquisition. 
A
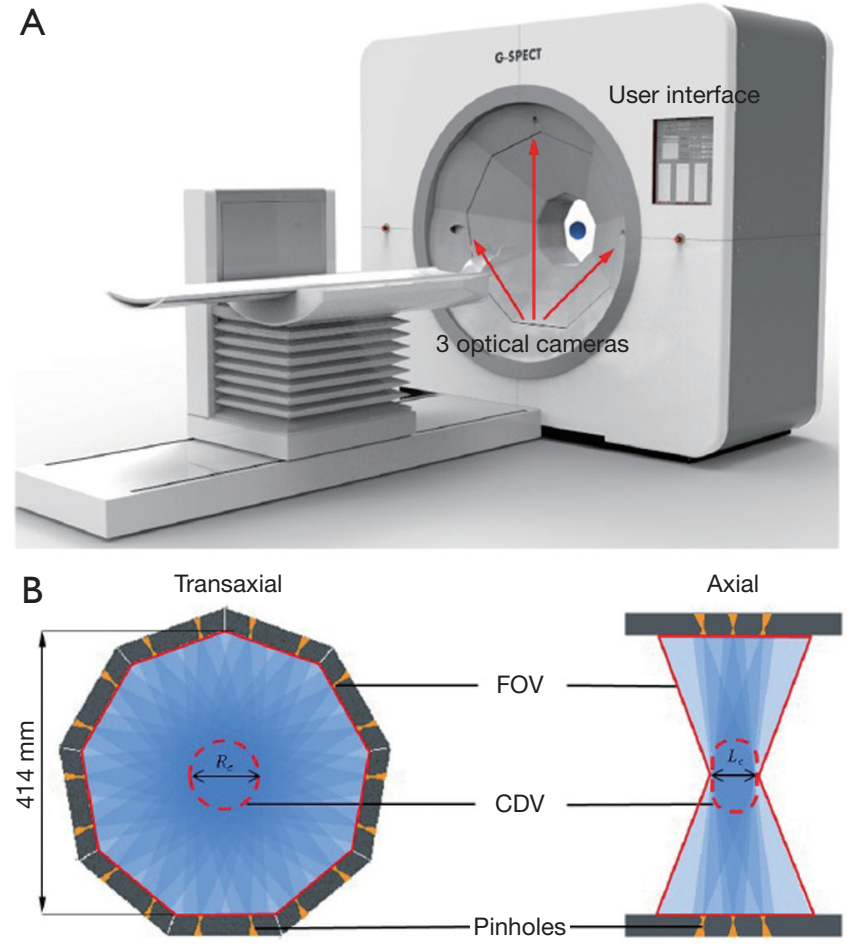

C

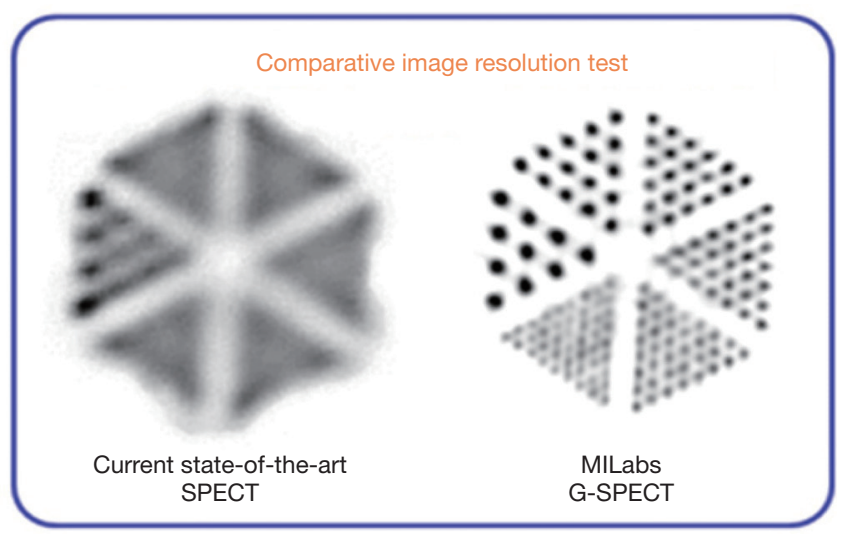

Figure 16 Illustration of a G-SPECT-I scanner. (A) G-SPECT-I system with three optical cameras and a user interface for volume-ofinterest (VOI) selection; (B) multi-pinhole collimator of G-SPECT-I system. All 54 pinholes distributed over three rings are focused on the complete data volume [CDV: the object shown in (A) in the FOV]. Each pinhole has an opening angle of $27^{\circ}$, resulting in a CDV with a transaxial diameter $\mathrm{R}_{c}$ of $100 \mathrm{~mm}$ and axial length $\mathrm{L}$ of $60 \mathrm{~mm}$ [courtesy of Chen et al. (82)]. (C) Comparison of resolution of G-SPECT-I equipped with a $38 \mathrm{~cm}$ collimator with a dual head Siemens Symbia system at equal scan time and equal dose based on physical experiments (with permission of MILabs B.V. Utrecht, The Netherlands).

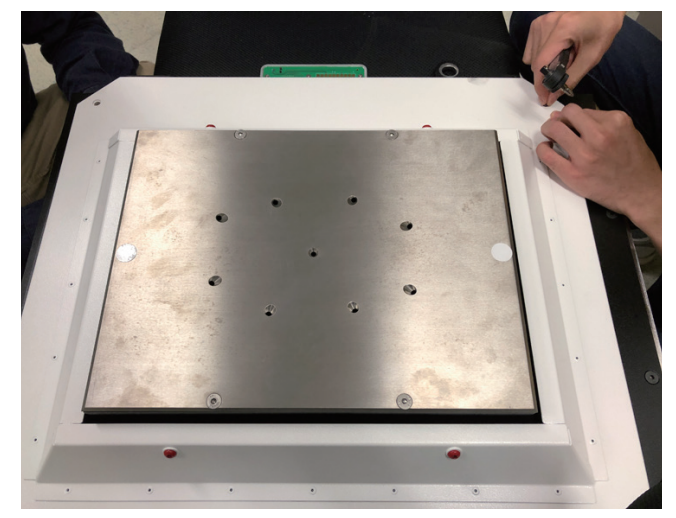

Figure 17 A sample 9-pinhole collimator (MPGP) mounted on a clinical SPECT scanner.

\section{Small organ MPH SPECT}

Bae et al. designed and evaluated an organ specific small
FOV MPH collimator (84). Their design consists of an 8 -pinhole collimator that can be integrated to a small FOV gamma camera, especially for thyroid imaging. Different from the regular cone shaped pinhole aperture, the pinholes were designed to be $2 \mathrm{~mm}$ diameter cylinders. Imaging distance and focal length were set at 150 and $50 \mathrm{~mm}$, respectively, resulting in a magnification of $1 / 3$. The pinholes were located as shown in Figure 19 and all of them were tilted towards the CFOV. The authors used two phantoms for evaluation. The first one is a twosphere phantom comprised of two spherical sources with a diameter of $5 \mathrm{~mm}$, one located at the CFOV and the other located $20 \mathrm{~mm}$ offset from the CFOV. The second one is a four-rod phantom comprises of four cylindrical rods that are located $30 \mathrm{~mm}$ offset from the center. The diameter of each rod is $5 \mathrm{~mm}$. Spatial resolutions were $6.8 \mathrm{~mm}$ and $7.6 \mathrm{~mm}$ for the two-sphere phantom and four-rod phantom, respectively. The system sensitivity was found to be 


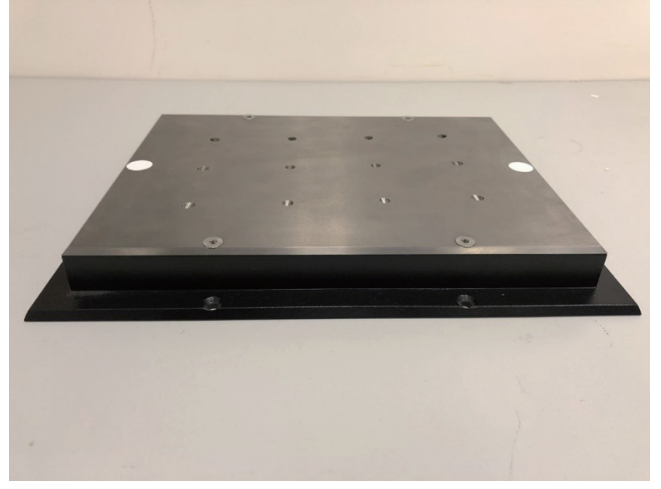

Figure 18 The 12-pinhole collimator plate for cardiac imaging.

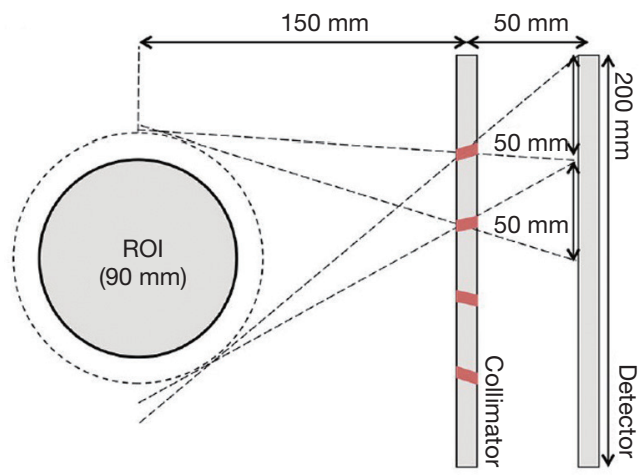

Figure 19 Proposed small organ MPH collimator [courtesy of Bae et al. (84)].

6.1 times higher than that of a single pinhole collimator.

\section{Robotic MPH SPECT}

A 49-pinhole SPECT system was designed and investigated by simulations for use as a computer-aided robotic system for head, breast, thorax, arms and legs imaging in radiation therapy (Figure 20) by Bowsher et al. (85). They assessed the feasibility of maneuvering the SPECT collimator system around the patient in position for radiation therapy, allowing an unrestricted therapy procedure. The system consists of 7 pods, i.e., one central pod and six peripheral pods, and each pod has a cluster of seven pinholes which target the same FOV. Each pinhole is coupled to a $7.6 \mathrm{~cm}$ wide circular detector with an active area of $7 \mathrm{~cm}$ in diameter. Pinhole-to-ROI distance ranges from 15 to $22 \mathrm{~cm}$ as the detector moves around the patient. The pinhole acceptance angle is $32.5^{\circ}$ and peripheral pods are tilted towards the central pod by $16.25^{\circ}$. Projections of an XCAT phantom representing a female patient in supine position were simulated. Reconstructed images of $7-\mathrm{mm}$ lesions in the breast region with 6:1 lesion-to-background ratio were obtained with $1.7 \mathrm{~mm}$ average localization error in as short as $4 \mathrm{~min}$.

\section{Molecular breast tomosynthesis (MBT)}

An MPH MBT system was proposed by van Roosmalen $e$ t al. for detecting breast tumors (11) that was then further developed and characterized by Wang et al. (86). In their designs, the patient lies prone on a specially designed bed with the scanner underneath. The pendant breast is compressed by transparent compression plates and imaged by two tungsten collimators with $10 \mathrm{~mm}$ thickness, each with 63 pinholes (Figure 21). The knife-edge pinholes have an acceptance angle of $42^{\circ}$ and a diameter of $2.7 \mathrm{~mm}$. A tungsten shielding plate was placed between the detector and the collimator to prevent multiplexing (50). The gamma camera used in the simulation employs a $\mathrm{NaI}(\mathrm{Tl})$ scintillation crystal with $3.5 \mathrm{~mm}$ intrinsic spatial resolution. In their work, they investigated whether the optimal collimator-detector geometry can improve the tumor-to-background ratio. They fixed the detector area and the distance between the breast and pinhole center at a minimum feasible value, while the pinhole diameter was calculated to reach the target system resolution. They also calculated the pinhole acceptance angle to keep the number of pinholes constant, and optimized sensitivity at several target system resolutions. The improved system can reach $3.5 \mathrm{~mm}$ resolution for a clinical $99 \mathrm{mTc}$ activity concentration in an 11-minute scan, while the smallest spheres visible in breast phantoms were $6 \mathrm{~mm}$ in diameter for the same scan time. The peak sensitivity can reach $0.37 \%$ for the focusing mode with a restricted FOV of $27 \mathrm{~mm}$. A whole breast can be scanned by 90 scan positions.

\section{Dynamic cardiac (DC) SPECT}

DC-SPECT was proposed and evaluated by Uzun Ozsahin et al. (87) for DC imaging studies. The system is based on simultaneous acquisitions of multiple projections from multiple detectors where each one is coupled with an individual pinhole (Figure 22). The main feature of the system is that the laser-induced optical barriers technique (87) is applied to the CsI:Tl crystal as opposed to physically cutting the crystals, which provides cost-effective scintillator-based detectors with a high intrinsic resolution. 

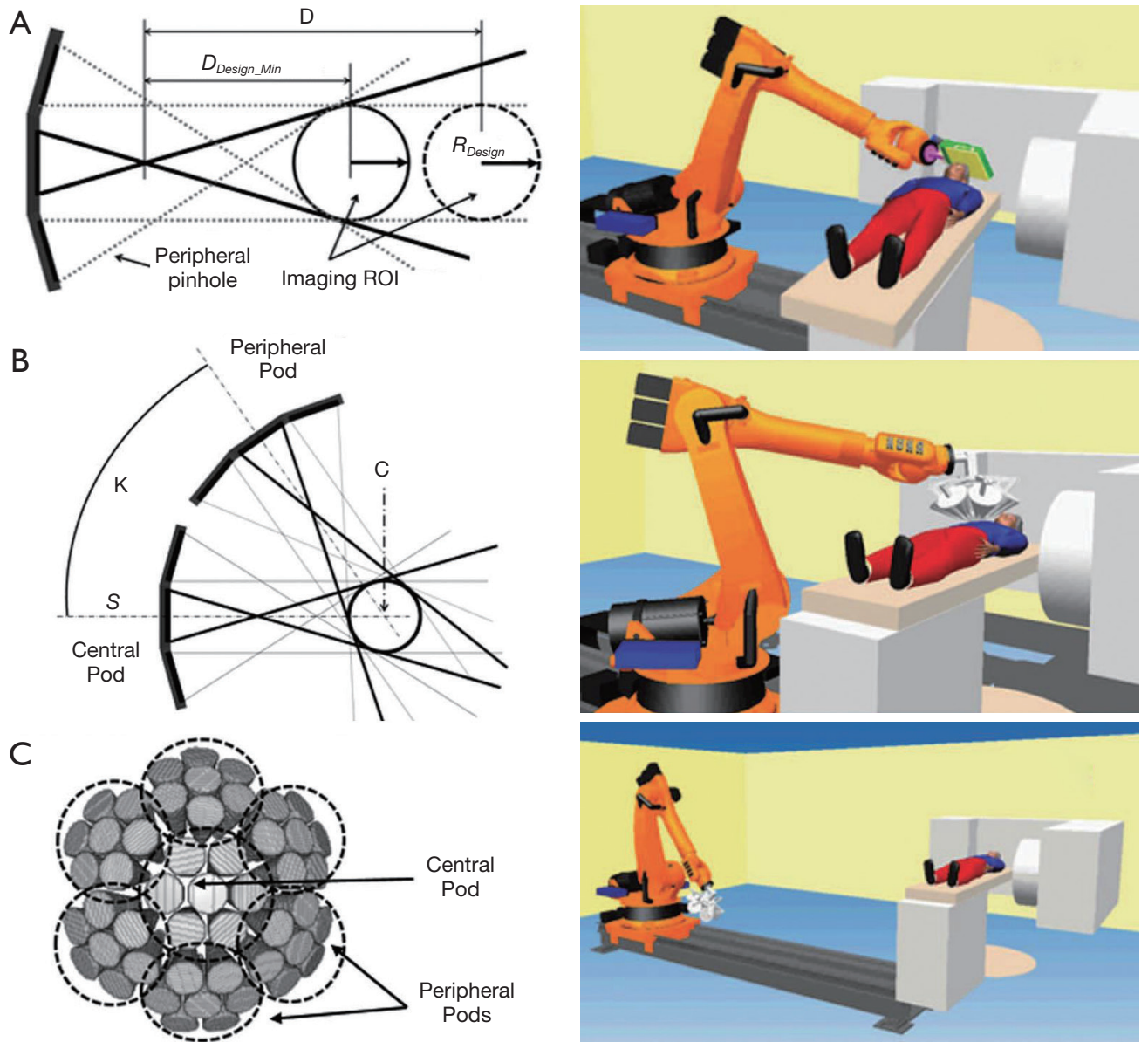

Figure 20 Left: (A) pod geometry. A planar cut through the central detector and two peripheral detectors from a seven-pinhole pod; (B) a planar cut through the central pod and one peripheral pod; (C) 3D rendering view of the 49-pinhole configuration. Right: representation of robotic SPECT imaging for a patient in position for radiation therapy. (A) Parallel-hole SPECT imaging of the head; (B) 49-pinhole SPECT imaging of the thorax; (C) robotic SPECT system retracted to allow normal external beam radiation therapy gantry motion [courtesy of Bowsher et al. (85)].

The authors considered the use of a multipixel photon counter, also known as silicon photomultiplier (SiPM), to read out the light from the fabricated arrays (88). Each detector module has an area of $70 \mathrm{~mm} \times 70 \mathrm{~mm}$ with a thickness of $10 \mathrm{~mm}$, pixelated into $2 \mathrm{~mm} \times 2 \mathrm{~mm}$ pixels and coupled to a single pinhole collimator. The hole diameter is $3 \mathrm{~mm}$ and the aperture-to-detector distance is set to $70 \mathrm{~mm}$. Each detector-pinhole combination focuses and covers the heart, which is modeled as an $18 \mathrm{~cm}$ diameter sphere (Figure 22). For comparison, they also simulated three commercially available scanners, i.e., D-SPECT (89), GE Discovery NM 530c (4) and conventional dual head GE Infinia. GEANT4 application for tomographic emission (GATE) (90) simulations for the four scanners were performed to evaluate the systems in terms of sensitivity and spatial resolution by using a point source placed at the CFOV. In addition, a Derenzo phantom was simulated for the proposed design to assess the image quality. The results showed that the proposed system has a spatial resolution of $10.0 \mathrm{~mm}$ and a sensitivity of $0.081 \%$, while they were $20.1 \mathrm{~mm}$ and $0.084 \%, 15.0 \mathrm{~mm}$ and $0.049 \%, 15.2 \mathrm{~mm}$ and $0.011 \%$ for the D-SPECT, GE Discovery NM 530c and conventional dual head GE Infinia, respectively.

\section{AdaptiSPECT-C}

AdaptiSPECT-C is a multi-detector MPH brain SPECT designed jointly by groups at UMass Medical School and 

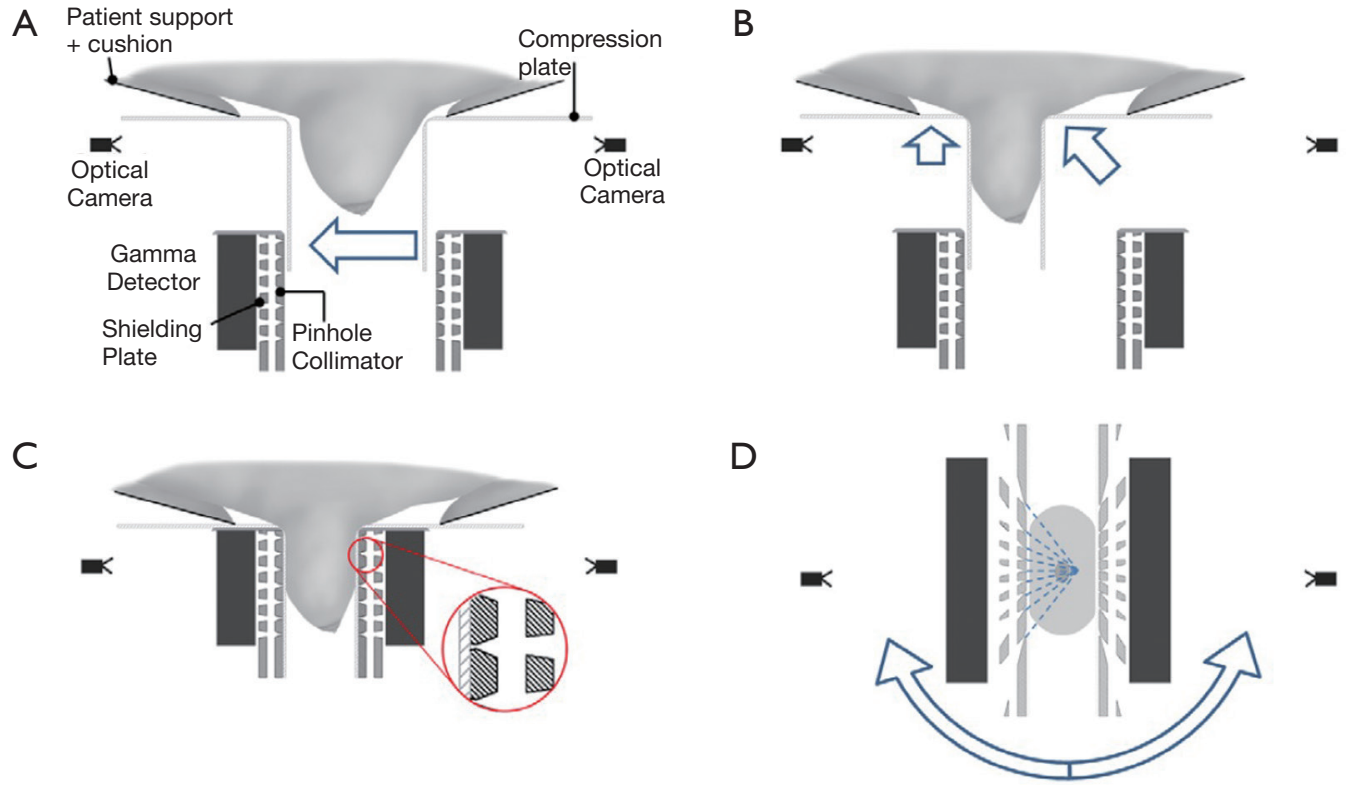

Figure 21 The compression process and scanner geometry for MBT. (A) Transparent plates compress the breast and a VOI is selected using optical cameras. (B) After the scan volume selection, the collimator plates and gamma detectors move into scanning position (arrows). (C) Collimator and gamma detector in scanning position, with insert showing collimator details. (D) Perpendicular cross-section through collimator-detector set-up showing the pinhole geometry. Dashed lines indicate the pinhole axes, which converge on a line 40 mm from the collimator. Arrows indicate the rotation of the whole scanner (including detectors, collimators and actuators) to enable acquisitions of different views [courtesy of van Roosmalen et al. (11)].

the University of Arizona (91) for clinical brain imaging with improved resolution and sensitivity. The system is being constructed at the University of Arizona. The preliminary prototype design for the system consisted of three closely packed rings of $0.95 \mathrm{~mm}$ thick hexagonal $\mathrm{NaI}(\mathrm{Tl})$ detectors (Figure 23). Specifications such as pinhole aperture size and number of pinholes open can be adjusted based on individual patients and applications (92). They evaluated the axial and angular sampling sufficiency of the proposed system with analytical simulations and reconstructions, using an XCAT digital brain phantom and a modified Defrise multi-disk phantom with a maximum radius of $9.2 \mathrm{~cm}$ and a disk pitch of $0.8 \mathrm{~cm}$ (93). Simulations were performed with the base configuration of a singlecentral pinhole per detector, and the usage of temporal shuttering to close that aperture and open two other apertures on either side of the central pinhole in the axial direction. Angular sampling of the base configuration was enhanced by adding the temporal shuttering of two additional apertures per detector positioned laterally to either side of the central aperture. The normalized mean square error (NMSE) was calculated for the reconstructed

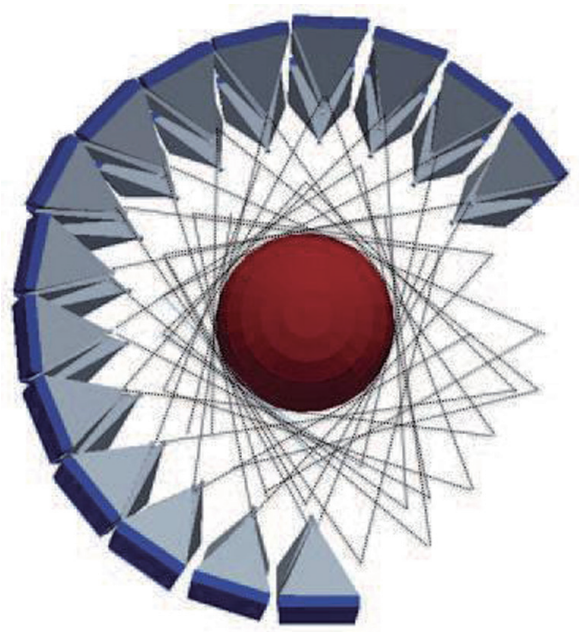

Figure 22 The 45 detector modules are arranged in 3 rows in the proposed dynamic MPH cardiac SPECT design [courtesy of Uzun Ozsahin et al. (87)].

images versus the original source distribution. The reconstructed images showed that increased axial sampling by combined reconstruction of acquisitions from solely 
the base apertures and then solely the two axially spaced apertures for Defrise phantom resulted in an increase in the number of disks resolved from 6 to 13 (all). Similarly, the reconstructed images showed that increasing angular sampling for the brain phantom resulted in reduced NMSE and better visualization of brain structures.

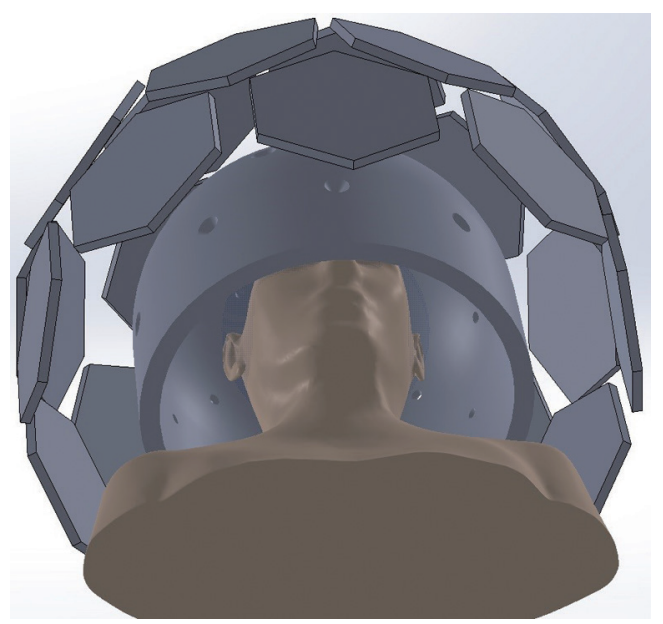

Figure 23 The preliminary prototype design of AdaptiSPECT-C together with a cropped XCAT phantom positioned inside. This design consists of three hexagonal detector rings: caudal ring with 9 detectors, middle ring with 9 detectors, and quasi-vertex ring with 5 detectors. Shown are the $2 \mathrm{~cm}$ thick aperture plate with a single aperture per detector and the 23 hexagonal detectors (courtesy of Dr. Kesava S. Kalluri).

\section{Triple-bead SPECT}

Ogawa et al. (94) developed a stationary triple-head MPH SPECT system for dynamic brain and cardiac studies. Different numbers of collimators and acquisition geometry were evaluated to find the best design. MPH collimators are tilted to cover the FOV. They evaluated 3 to 12 knife-edge pinholes for each camera with no multiplexing (Figure 24). Each camera has an active area of $400 \mathrm{~mm} \times 200 \mathrm{~mm}$. The distance from the detector surface to the CFOV is $30 \mathrm{~cm}$ and $27 \mathrm{~cm}$ for brain and cardiac imaging, respectively. The pinhole diameter was defined as $3 \mathrm{~mm}$, with a collimator plate thickness of $10 \mathrm{~mm}$. Point source, brain and cardiac phantoms were simulated and images were reconstructed with OS-EM algorithm. CT was used for attenuation correction. They found that at least five pinholes are required to adequately reconstruct the point sources, and FWHMs of the MPH collimators are smaller than that of the parallel-hole collimator, i.e., 9-11 $\mathrm{mm}$ (depending on the number of the pinholes) versus $12 \mathrm{~mm}$. The SNR (defined by mean/standard deviation) of MPH collimators was found to be much worse compared to that of the parallel-hole collimator, i.e., 10 versus 60 . For the brain phantom, the image quality improves as the number of the pinholes increases and at least eight pinholes are required to generate images without significant artifacts. They also showed that increasing the number of pinholes results in a decreased mean squared error (MSE) between the original phantom and reconstructed image. If the number

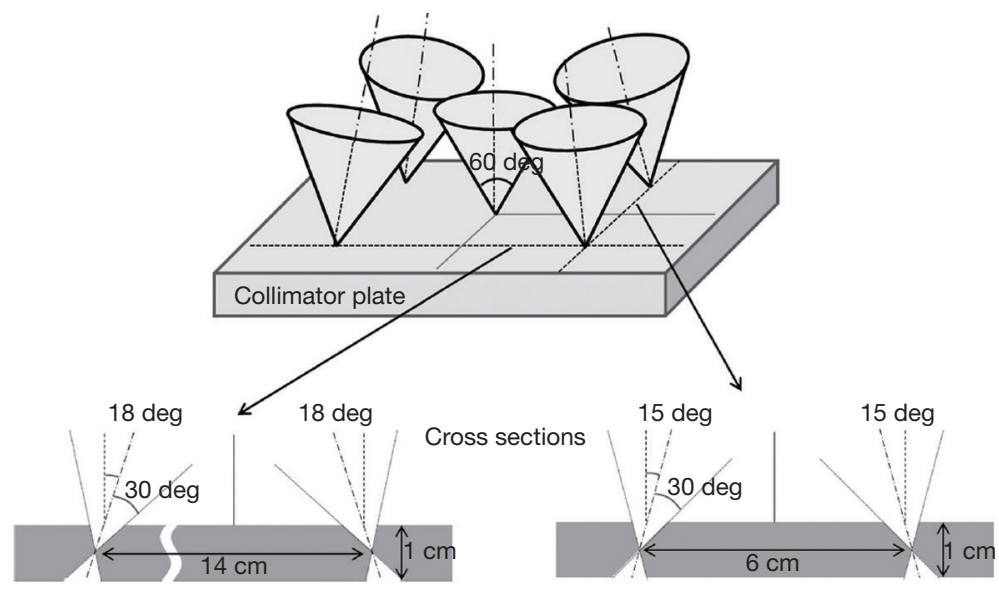

Figure 24 Five pinholes are located on a pinhole plate for the triple-head SPECT system. The thickness of the pinhole plate is $1 \mathrm{~cm}$ and each pinhole has a knife edge at the half depth of the pinhole plate. The axis of each pinhole has a different angle [courtesy of Ogawa $\mathrm{et}$ al. (94)]. 


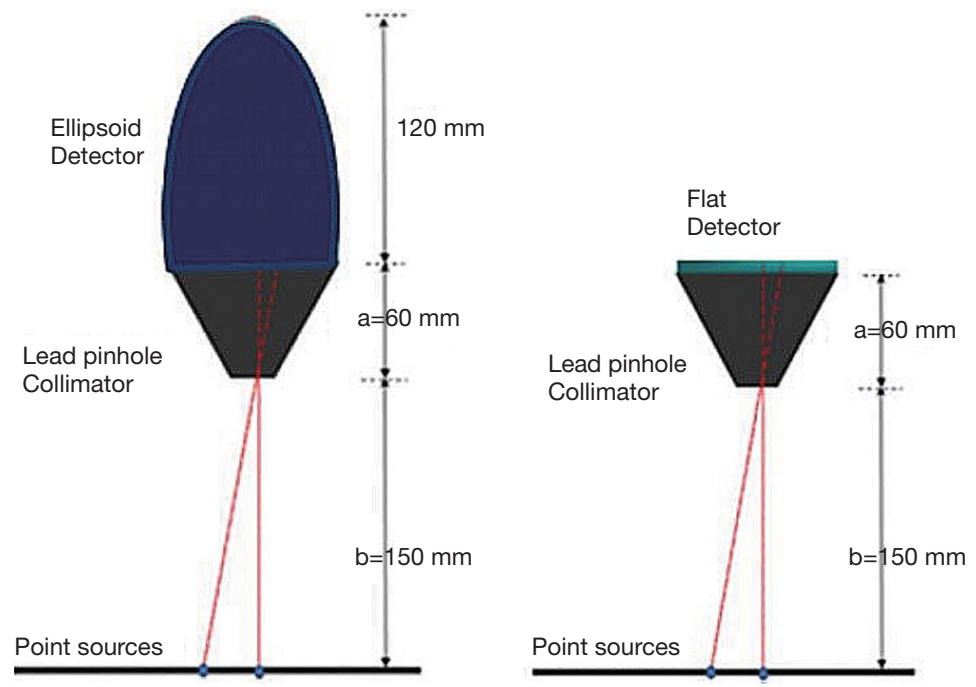

Figure 25 GATE simulation setup using (A) ellipsoid detector and (B) flat detector, with point-sources located at $150 \mathrm{~mm}$ from the pinhole aperture [courtesy of Bhusal et al. (95)].

of pinholes is eight or higher, the MSE reaches a plateau with a value similar to that of the parallel-hole collimator. Myocardial phantom simulation results showed that with more than eight pinholes for each detector, one can obtain similar image quality to that of the parallel hole collimator.

\section{Hemi-ellipsoid SPECT}

Bhusal et al. (95) investigated a novel cardiac SPECT system consisting of 21 pinhole collimators, each coupled to a hemi-ellipsoid detector. They performed GATE simulations with various phantoms to evaluate the system performance. Left ventricle counts were used to evaluate the system sensitivity and then compared to a state-ofthe-art scanner (89). The system employs ellipsoidal CsI scintillation crystals with $6 \mathrm{~mm}$ thickness, $80 \mathrm{~mm}$ diameter and $120 \mathrm{~mm}$ height (Figure 25). The detector configuration is based on the original design proposed by Dey (96) and further improved by Kalluri et al. (97). Spatial resolution was found to be $4.5 \mathrm{~mm}$ and the sensitivity was 3.4 times higher $(0.289 \%)$ than that of D-SPECT. They also showed that the increased sensitivity can be used for an ultra-low dose $(111 \mathrm{MBq})$ acquisition with $5.4 \mathrm{~min}$ scan time and the results are comparable to those of the full dose (925 MBq) acquisition with 2 min scan time of D-SPECT.

\section{MPH SPECT/MRI}

Even though SPECT/CT hybrid systems have been available for nearly two decades, the combination of a SPECT system with MRI is still in progress due to technical challenges including the need for MRIcompatible components of SPECT and driving applications. The first step to develop a clinical SPECT/ MRI system is similar to what has been done for PET/ MRI, where a PET insert was designed to be utilized with an existing MRI system. Accomplishing this for SPECT/MRI is still being researched for clinical brain imaging (98-100). Conventional photomultiplier tubes (PMTs) should be replaced with SiPMs when designing a SPECT/MRI system, not only due to the fact that PMTs are affected by the strong magnetic field of MRI, but also due to the space constraints of the MRI bore, especially when considering the collimators for SPECT. Busca et al. (99) designed a clinical MPH SPECT/MRI system to image the whole brain (Figure 26). The proposed design consists of two detector rings with $35 \mathrm{~cm}$ in diameter and is inserted into a $3 \mathrm{~T}$ MRI system with $59 \mathrm{~cm}$ bore size. The system resolution was set to $8-10 \mathrm{~mm}$, which is similar to that of a brain SPECT scanner and the number of detectors was arranged to maximize the sensitivity (0.018-0.032\%) (98). According to the simulation results, 


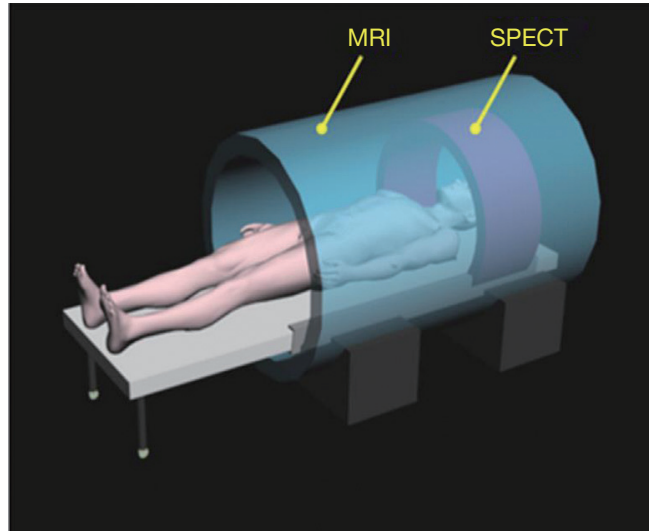

Figure 26 Proposed design of the integrated SPECT/MRI instrument (courtesy of Busca et al. (100)]. the diameter of FOV was determined as $18-20 \mathrm{~cm}$ while the number of detector modules was $27-30$.

\section{Summary of discussed system characteristics}

A summary of the characteristics for the different clinical MPH SPECT systems mentioned is listed in Table 1. Note that for G-SPECT, FASTSPECT and many other MPH SPECT systems, fast SPECT (sub-second) acquisitions are inherently feasible.

\section{Conclusions}

Cardiovascular disease is the greatest cause of mortality and morbidity in the world and SPECT is one of the

Table 1 Summary of the clinical MPH SPECT systems

\begin{tabular}{|c|c|c|c|c|c|c|c|}
\hline System name & $\begin{array}{l}\text { Resolution } \\
(\mathrm{mm}) / \text { imaging } \\
\text { distance }(\mathrm{mm})\end{array}$ & $\begin{array}{l}\text { Reported } \\
\text { Sensitivity }\end{array}$ & $\begin{array}{c}\text { Calculated } \\
\text { sensitivity (\%) }\end{array}$ & $\begin{array}{l}\text { FOV (in diameter, } \\
\text { mm) }\end{array}$ & VOI & Isotope & Ref. \\
\hline 7-pinhole Anger camera & $10 / 127$ & $750 \mathrm{cps} / \mathrm{MBq}$ & 0.075 & 127 & Heart & ${ }^{201} \mathrm{Tl}$ & $(33,76)$ \\
\hline FASTSPECT & $4.8 / 100$ & $973.0 \mathrm{cps} / \mathrm{MBq}$ & 0.097 & $\begin{array}{c}200 \times 200 \times 100 \\
\text { ellipsoid }\end{array}$ & Brain & ${ }^{99 \mathrm{~m}} \mathrm{Tc}$ & $(77,78)$ \\
\hline MPH MPI SPECT & $13 / 100$ & $\begin{array}{l}5 \times 10^{6} \text { (a.u.) (10-fold } \\
\text { higher than LEGP) }\end{array}$ & 0.119 & $\mathrm{~N} / \mathrm{A}$ & Heart & ${ }^{201} \mathrm{Tl}$ & (64) \\
\hline Discovery NM 530c & $6.1 / 100$ & $640 \mathrm{cps} / \mathrm{MBq}$ & 0.064 & $\begin{array}{c}\text { ellipsoid with } \\
\text { dimensions 190 }\end{array}$ & Heart & $\begin{array}{l}{ }^{99 \mathrm{~m}} \mathrm{Tc}, \\
{ }^{201} \mathrm{TI},{ }^{123} \mathrm{I}\end{array}$ & $(4,79)$ \\
\hline Multi-lofthole brain SPECT & $6.3 / 145$ & $155 \mathrm{cps} / \mathrm{MBq}$ & 0.016 & $\begin{array}{l}220 \text { (124 mm } \\
\text { long cylinder }\end{array}$ & Brain & ${ }^{99 \mathrm{~m}} \mathrm{Tc}$ & (81) \\
\hline 20-pinhole SPECT & 20.6/193 & $0.160 \%$ & 0.160 & 210 & Brain \& heart & ${ }^{123} \mathrm{I},{ }^{99 \mathrm{~m}} \mathrm{TC}$ & $(3,5)$ \\
\hline 9-pinhole brain SPECT & $12.0 \& 8.0 / 167$ & $0.037 \% \& 0.014 \%$ & $0.037 \& 0.014$ & 200 & Brain & ${ }^{9 \mathrm{~m}} \mathrm{Tc}$ & (10) \\
\hline $\begin{array}{l}\text { Multi-pinhole cardiac } \\
\text { SPECT (12-pinhole \& } \\
\text { 14-pinhole) }\end{array}$ & $\begin{array}{c}10.0 / 186 \& \\
8.2 / 220\end{array}$ & $0.035 \% \& 0.017 \%$ & $0.035 \& 0.017$ & $160 \& 200$ & Heart & ${ }^{9 \mathrm{~m}} \mathrm{Tc}$ & $(8,9)$ \\
\hline Small organ MPH SPECT & $6.8-7.6$ & $\mathrm{~N} / \mathrm{A}$ & $\mathrm{N} / \mathrm{A}$ & 90 & $\begin{array}{l}\text { Small } \\
\text { organs }\end{array}$ & ${ }^{123} \mid$ & (84) \\
\hline Robotic MPH SPECT & $\begin{array}{l}7 \mathrm{~mm} \text { lesions } \\
\text { resolved }\end{array}$ & $\mathrm{N} / \mathrm{A}$ & $\mathrm{N} / \mathrm{A}$ & 83 & $\begin{array}{l}\text { Small } \\
\text { organs }\end{array}$ & ${ }^{9 \mathrm{~m}} \mathrm{Tc}$ & (85) \\
\hline MBT & 3.5 & $0.082-0.370 \%$ & $0.082-0.370 \%$ & 27 & Breast & ${ }^{9 \mathrm{~m}} \mathrm{Tc}$ & $(11,86)$ \\
\hline DC-SPECT & 10 & $0.081 \%$ & 0.081 & 180 & Heart & ${ }^{9 \mathrm{~m}} \mathrm{Tc}$ & (87) \\
\hline
\end{tabular}

*, body collimator is under characterization. 
most established imaging methods to assess myocardial perfusion. MPH cardiac SPECT significantly reduces the acquisition time, thus increasing the patient throughput and improving the patient comfort compared to the use of a conventional parallel hole collimator. This increasing demand has led to the development of several dedicated commercial MPH SPECT scanners for cardiac applications that are currently on the market, with various research prototypes developed or being investigated. On the other hand, along with the existing cerebral blood flow tracers, new brain SPECT tracers for Alzheimer's and Parkinson's diseases are either already available as in the case of I-123 labeled DaTscan (101), or are on the horizon, such as Tau imaging (102). To facilitate early diagnosis for potential treatment, the development of high-resolution brain SPECT is in demand. Thus, clinical MPH SPECT systems are currently being developed for brain and cardiac imaging for the most part, which target relatively small or medium sized VOIs that are suitable for pinhole imaging. The potential of MPH SPECT imaging for other applications, e.g., breast, thyroid and pediatric applications is being explored. The use of MPH collimators plays a significant role in improving current SPECT performance, and will remain a subject of interest for researchers as well as commercially with regard to the development of new SPECT systems in the future.

\section{Acknowledgments}

Funding: This work was supported by a research grant from Macau Science and Technology Development Fund (0091/2019/A2).

\section{Footnote}

Conflicts of Interest: All authors have completed the ICMJE uniform disclosure form (available at http://dx.doi. org/10.21037/qims-19-1036). FJB reports personal fees and non-financial support from MILabs, grants from Dutch Foundation for Scientific Research (NWO), during the conduct of the study. In addition, FJB patents on several pinhole imaging devices issued. He is founder, shareholder, and part time CEO of MILabs B.V, a company that develops pinhole PET and SPECT systems like the U-SPECT and G-SPECT scanners that were addressed in the present paper. GSPM reports that she has a patent "Optimized Multi-Pinhole Collimator for Dual-Purpose Clinical and Preclinical Imaging" (US 9,431,140 B2) issued.
In addition, GSPM serves as an unpaid editorial board member of Quantitative Imaging in Medicine and Surgery. The other authors have no conflicts of interest to declare.

Open Access Statement: This is an Open Access article distributed in accordance with the Creative Commons Attribution-NonCommercial-NoDerivs 4.0 International License (CC BY-NC-ND 4.0), which permits the noncommercial replication and distribution of the article with the strict proviso that no changes or edits are made and the original work is properly cited (including links to both the formal publication through the relevant DOI and the license). See: https://creativecommons.org/licenses/by-nc-nd/4.0/.

\section{References}

1. Beekman F, van der Have F. The pinhole: gateway to ultrahigh-resolution three-dimensional radionuclide imaging. Eur J Nucl Med Mol Imaging 2007;34:151-61.

2. Furenlid LR, Wilson DW, Chen YC, Kim H, Pietraski PJ, Crawford MJ, Barrett HH. FastSPECT II: a secondgeneration high-resolution dynamic SPECT imager. IEEE Trans Nucl Sci 2004;51:631-5.

3. Bowen JD, Huang Q, Ellin JR, Lee TC, Shrestha U, Gullberg GT, Seo Y. Design and performance evaluation of a 20 -aperture multipinhole collimator for myocardial perfusion imaging applications. Phys Med Biol 2013;58:7209-26.

4. Bocher M, Blevis IM, Tsukerman L, Shrem Y, Kovalski G, Volokh L. A fast cardiac gamma camera with dynamic SPECT capabilities: design, system validation and future potential. Eur J Nucl Med Mol Imaging 2010;37:1887-902.

5. Lee TC, Ellin JR, Huang Q, Shrestha U, Gullberg GT, Seo Y. Multipinhole collimator with 20 apertures for a brain SPECT application. Med Phys 2014;41:112501.

6. Beekman FJ, van der Have F, Goorden MC, Vaissier PEB, van Roosmalen J, During H. G-SPECT-I: a full ring high sensitivity and ultra-fast clinical molecular imaging system with $<3 \mathrm{~mm}$ resolution. Eur J Nucl Med Mol Imaging 2015;42:S209.

7. King MA, Mukherjee JM, Konik A, Zubal IG, Dey J, Licho R. Design of a multi-pinhole collimator for I-123 DaTscan imaging on dual-headed SPECT systems in combination with a fan-beam collimator. IEEE Trans Nucl Sci 2016;63:90-7.

8. Si C, Mok GS, Chen L, Tsui BM. Design and evaluation of an adaptive multipinhole collimator for high-performance clinical and preclinical imaging. Nucl Med Commun 
2016;37:313-21.

9. Yan PY, Chen L, Tsui BMW, Mok GSP. Evaluation of stationary and semi-stationary acquisitions in a dual-head multi-pinhole collimator for myocardial perfusion SPECT. J Med Biol Eng 2016;36:675-85.

10. Chen L, Tsui BM, Mok GS. Design and evaluation of two multi-pinhole collimators for brain SPECT. Ann Nucl Med 2017;31:636-48.

11. van Roosmalen J, Goorden MC, Beekman FJ. Molecular breast tomosynthesis with scanning focus multi-pinhole cameras. Phys Med Biol 2016;61:5508.

12. McDougal F, Tornai M. Molecular breast imaging using a fully tomographic stationary clinical cardiac SPECT scanner: a phantom study. J Nucl Med 2017;58:1364.

13. Slomka PJ, Patton JA, Berman DS, Germano G. Advances in technical aspects of myocardial perfusion SPECT imaging. J Nucl Cardiol 2009;16:255-76.

14. Steele PP, Kirch DL, Koss JE. Comparison of simultaneous dual-isotope multipinhole SPECT with rotational SPECT in a group of patients with coronary artery disease. J Nucl Med 2008;49:1080-9.

15. Ben-Haim S, Murthy VL, Breault C, Allie R, Sitek A, Roth N, Fantony J, Moore SC, Park MA, Kijewski M, Haroon A, Slomka P, Erlandsson K, Baavour R, Zilberstien Y, Bomanji J, Di Carli MF. Quantification of myocardial perfusion reserve using dynamic SPECT imaging in humans: a feasibility study. J Nucl Med 2013;54:873-9.

16. Shrestha U, Sciammarella M, Alhassen F, Yeghiazarians $Y$, Ellin J, Verdin E, Boyle A, Seo Y, Botvinick EH, Gullberg GT. Measurement of absolute myocardial blood flow in humans using dynamic cardiac SPECT and 99mTctetrofosmin: Method and validation. J Nucl Cardiol 2017;24:268-77.

17. Matsuda H. Role of neuroimaging in Alzheimer's disease, with emphasis on brain perfusion SPECT. J Nucl Med 2007;48:1289-300.

18. Yeo JM, Lim X, Khan Z, Pal S. Systematic review of the diagnostic utility of SPECT imaging in dementia. Eur Arch Psychiatry Clin Neurosci 2013;263:539-52.

19. Davison CM, O'Brien J T. A comparison of FDGPET and blood flow SPECT in the diagnosis of neurodegenerative dementias: A systematic review. Int J Geriatr Psychiatry 2014;29:551-61.

20. Nemoto K. Brain perfusion SPECT in Alzheimer's disease. In: Matsuda H, Asada T, Tokumaru A. (eds) Neuroimaging diagnosis for Alzheimer's disease and other dementias. Tokyo: Springer, 2017.

21. Van Patten R, Greif T, Britton K, Tremont G. Single- photon emission computed tomography (SPECT) perfusion and neuropsychological performance in mild cognitive impairment. J Clin Exp Neuropsychol 2019;41:530-43.

22. Mozley PD, Schneider JS, Acton PD, Plössl K, Stern MB, Siderowf A, Leopold NA, Li PY, Alavi A, Kung HF. Binding of [99m Tc]TRODAT-1 to dopamine transporters in patients with Parkinson's disease and in healthy volunteers. J Nucl Med 2000;41:584-9.

23. Huang WS, Lin SZ, Lin JC, Wey SP, Ting G, Liu RS. Evaluation of early-stage Parkinson's disease with 99mTcTRODAT-1 imaging. J Nucl Med 2001;42:1303-8.

24. Chou KL, Hurtig HI, Stern MB, Colcher A, Ravina B, Newberg A, Mozley PD, Siderowf A. Diagnostic accuracy of $[(99 \mathrm{~m})$ Tc $]$ TRODAT-1 SPECT imaging in early Parkinson's disease. Parkinsonism Relat Disord 2004;10:375-9.

25. Kägi G, Bhatia KP, Tolosa E. The role of DAT-SPECT in movement disorders. J Neurol Neurosurg Psychiatry 2010;81:5-12.

26. Wanet PM, Sand A, Abramovici J. Physical and clinical evaluation of high-resolution thyroid pinhole tomography. J Nucl Med 1996;37:2017-20.

27. Tomas MB, Pugliese PV, Tronco GG, Love C, Palestro CJ, Nichols KJ. Pinhole versus parallel-hole collimators for parathyroid imaging: An intraindividual comparison. J Nucl Med Technol 2008;36:189-94.

28. Keidar Z, Israel O, Krausz Y. SPECT/CT in tumor imaging: technical aspects and clinical applications. Semin Nucl Med 2003;33:205-18.

29. Gupta A, Kim KY, Hwang D, Lee MS, Lee DS, Lee JS. Performance evaluation and quantitative accuracy of multipinhole nanoSPECT/CT scanner for theranostic Lu177 imaging. J Korean Phys Soc 2018;72:1379-86.

30. Van Audenhaege K, Van Holen R, Vandenberghe S, Vanhove C, Metzler SD, Moore SC. Review of SPECT collimator selection, optimization, and fabrication for clinical and preclinical imaging. Med Phys 2015;42:4796-813.

31. Gullberg GT, Zeng GL, Datz FL, Christian PE, Tung $\mathrm{CH}$, Morgan HT. Review of convergent beam tomography in single photon emission computed tomography. Phys Med Biol 1992;37:507-34.

32. Jaszczak RJ, Li J, Wang H, Zalutsky MR, Coleman RE. Pinhole collimation for ultra-high-resolution, small-fieldof-view SPECT. Phys Med Biol 1994;39:425.

33. Vogel RA, Kirch D, LeFree M, Steele P. A new method of multiplanar emission tomography using a seven pinhole 
collimator and an Anger scintillation camera. J Nucl Med 1978;19:648-54.

34. Vanhove C, Defrise M, Lahoutte T, Bossuyt A. Threepinhole collimator to improve axial spatial resolution and sensitivity in pinhole SPECT. Eur J Nucl Med Mol Imaging 2008;35:407-15.

35. Difilippo FP. Design and performance of a multi-pinhole collimation device for small animal imaging with clinical SPECT and SPECT-CT scanners. Phys Med Biol 2008;53:4185.

36. Mok GS, Yu J, Du Y, Wang Y, Tsui BM. Evaluation of a multi-pinhole collimator for imaging small animals with different sizes. Mol Imaging Biol 2012;14:60-9.

37. Könik A, Auer B, De Beenhouwer J, Kalluri K, Zeraatkar N, Furenlid LR, King MA. Primary, scatter, and penetration characterizations of parallel-hole and pinhole collimators for I-123 SPECT. Phys Med Biol 2019;64:245001.

38. Smith MF, Jaszczak RJ, Wang H, Li J. Lead and tungsten pinhole inserts for I-131 SPECT tumor imaging: experimental measurements and photon transport simulations, IEEE Trans Nucl Sci 1997;44:74-82.

39. Hubbell JH. Photon cross sections, attenuation coefficients, and energy absorption coefficients from 10 $\mathrm{keV}$ to $100 \mathrm{GeV} 1969$ National Bureau of Standards (U.S.) Rep. NSRDS-NBS 29. Available online: https://srd.nist. gov/NSRDS/NSRDS-NBS29.pdf

40. Tornai MP, Pieper BC, Bowsher JE, Jaszczak RJ. Effects of pinhole material and aperture size on lesion contrast and SNR breast SPECT. Lyon, France: 2000 IEEE Nuclear Science Symposium. Conference Record (Cat. No.00CH37149), 2000.

41. Nguyen MP, Goorden MC, Kamphuis C, Beekman FJ. Evaluation of pinhole collimator materials for micronresolution ex vivo SPECT. Phys Med Biol 2019;64:105017.

42. Bal G, Acton PD. Analytical derivation of the point spread function for pinhole collimators. Phys Med Biol 2006;51:4923-50.

43. Metzler SD, Bowsher JE, Smith MF, Jaszczak RJ. Analytic determination of pinhole collimator sensitivity with penetration. IEEE Trans Med Imaging 2001;20:730-41.

44. Smith M, Jaszczak R, Wang H. Pinhole aperture design for 131I tumor imaging. IEEE Trans Nucl Sci 1997;44:1154-60.

45. Goorden MC, van der Have F, Kreuger R, Ramakers RM, Vastenhouw B, Burbach JP, Booij J, Molthoff CF, Beekman FJ. VECTor: a preclinical imaging system for simultaneous submillimeter SPECT and PET. J Nucl
Med 2013;54:306-12.

46. Beekman FJ, inventor; MILabs BV, assignee. Focused pinhole gamma detection device. U.S. patent 8,067,741. November 29, 2011. European Patent Application EP2073039.

47. Wang Y. Development and application of highsensitivity and high-resolution fully 3D SPECT imaging techniques using two different collimator designs. Doctor Dissertation. Chapel Hill: The University of North Carolina, 2004.

48. Huang PC, Hsieh HH, Hsu CH, Hsiao IT. An Efficient Sensitivity Calculation of Tilted Apertures for Preclinical Multi-pinhole SPECT. Biomed Eng: Appl Basis Commun 2015;27:1.

49. Paix D. Pinhole imaging of gamma rays. Phys Med Biol 1967;12:489-500.

50. Beekman FJ, van der Have F, Vastenhouw B, van der Linden AJ, van Rijk PP, Burbach JP, Smidt MP. U-SPECT-I: a novel system for submillimeter-resolution tomography with radiolabeled molecules in mice. J Nucl Med 2005;46:1194-200.

51. van der Have F, Vastenhouw B, Ramaker RM, Branderhorst W, Krah JO, Ji C, Staelens SG, Beekman FJ. U-SPECT-II: an ultra-high-resolution device for molecular small-animal imaging J Nucl Med 2009;50:599-605.

52. Deprez K, Pato LR, Vandenberghe S, Van Holen R. Characterization of a SPECT pinhole collimator for optimal detector usage (the lofthole). Phys Med Biol 2013;58:859.

53. Metzler SD, Moore SC, Park MA, Design of a new smallanimal SPECT system based on rectangular pinhole aperture. Anaheim, CA, USA: 2012 IEEE NSS-MIC (M21-43), 2012.

54. Xia D, Moore SC, Park MA, Cervo M, Metzler SD. Investigation of imaging properties for submillimeter rectangular pinholes. Med Phys 2015;42:6933-44.

55. Moore SC, Park MA, Liu Z, Lyon MC, Johnson LC, Lushear VH, Westberg JG, Metzler SD. Design of a dualresolution collimator for preclinical cardiac SPECT with a stationary triple-detector system. Med Phys 2016;43:6336.

56. Mok GS, Tsui BM, Beekman FJ. The effects of object activity distribution on multiplexing multi-pinhole SPECT. Phys Med Biol 2011;56:2635.

57. Mok GS, Wang Y, Tsui BM. Quantification of the multiplexing effects in multi-pinhole small animal SPECT: a simulation study. IEEE Trans Nucl Sci 2009;56:2636-43.

58. Vunckx K, Suetens P, Nuyts J. Effect of overlapping projections on reconstruction image quality in 
multipinhole SPECT. IEEE Trans Med Imaging 2008;27:972-83.

59. Mahmood ST, Erlandsson K, Cullum I, Hutton BF. The potential for mixed multiplexed and non-multiplexed data to improve the reconstruction quality of a multi-slit-slat collimator SPECT system. Phys Med Biol 2010;5 5:2247.

60. Van Audenhaege K, Vanhove C, Vandenberghe S, Van Holen R. The evaluation of data completeness and image quality in multiplexing multi-pinhole SPECT. IEEE Trans Med Imaging 2015;34:474-86.

61. Kench PL, Lin J, Gregoire MC and Meikle SR. An investigation of inconsistent projections and artefacts in multi-pinhole SPECT with axially aligned pinholes. Phys Med Biol 2011;56:7487-503.

62. Moore SC, Cervo M, Metzler SD, Udias JM, Herraiz JL. An iterative method for eliminating artifacts from multiplexed data in pinhole SPECT. The 13th international meeting on fully three-dimensional image reconstruction in radiology and nuclear medicine, 2015;515-517.

63. Vaissier PE, Goorden MC, Vastenhouw B, van der Have F, Ramakers RM, Beekman FJ. Fast spiral SPECT with stationary gamma-cameras and focusing pinholes. J Nucl Med 2012;53:1292-9.

64. Funk T, Kirch DL, Koss JE, Botvinick E, Hasegawa BH. A novel approach to multipinhole SPECT for myocardial perfusion imaging. J Nucl Med 2006;47:595-602.

65. Mok GSP, Tsui BMW, Wang Y, Du Y, Segars WP, Frey EC. Effects of pinhole pattern and multiplexing in multipinhole small animal SPECT. 52nd Annual Meeting of the Society of Nuclear Medicine, 2005.

66. Hwang AB, Iwata K, Hasegawa BH, editors. Simulation of depth of interaction effects for pinhole SPECT. Lyon, France: IEEE Nuclear Science Symposium Conference Record, 2001.

67. Beekman FJ, Vastenhouw B. Design and simulation of a high-resolution stationary SPECT system for small animals. Phys Med Biol 2004;49:4579.

68. Korevaar MA, Heemskerk JW, Beekman FJ. A pinhole gamma camera with optical depth-of-interaction elimination. Phys Med Biol 2009;54:N267-72.

69. Feng B, Chen M, Bai B, Smith AM, Austin DW, Mintzer RA, Osborne D, Gregor J. Modeling of the point spread function by numerical calculations in single-pinhole and multipinhole SPECT reconstruction. IEEE Trans Nucl Sci 2010;57:173-80.

70. Wernick MN, Aarsvold JN. Emission tomography: the fundamentals of PET and SPECT. Academic Press, 2004.
71. Aguiar P, Pino F, Silva-Rodriguez J, Pavia J, Ros D, Ruibal A, El Bitar Z. Analytical, experimental, and Monte Carlo system response matrix for pinhole SPECT reconstruction. Med Phys 2014;41:032501.

72. Peterson M, Gustafsson J, Ljungberg M. Monte Carlobased quantitative pinhole SPECT reconstruction using a ray-tracing back-projector. EJNMMI Phys 2017;4:32.

73. Ljungberg M. In: Ljungberg M, Strand SE, King MA, editors. Monte Carlo calculations in nuclear medicine: Applications in diagnostic imaging, second edition. Francis \& Taylor, 2012:111-28.

74. Gieles M, de Jong H W, Beekman FJ. Monte Carlo simulations of pinhole imaging accelerated by kernel-based forced detection. Phys Med Biol 2002;47:1853-67.

75. Yao R, Ma T, Shao Y. Derivation of system matrix from simulation data for an animal SPECT with slit-slat collimator. IEEE T Nucl Sci 2009;56:2651-8.

76. LeFree MT, Vogel RA, Kirch DL, Steele PP. 7-pinhole tomography - a technical description. J Nucl Med 1981;22:48-54.

77. Rowe RK, Aarsvold JN, Barrett HH, Chen JC, Klein WP, Moore BA, Pang IW, Patton DD, White TA. A stationary hemispherical SPECT imager for three-dimensional brain imaging. J Nucl Med 1993;34:474-80.

78. Klein WP, Barrett H, Pang IW, Patton DD, Rogulski MM, Sain J, Smith W. FASTSPECT: electrical and mechanical design of a high-resolution dynamic SPECT imager. Lyon, France: IEEE NSS/MIC Conf. Record 1995;2:931-3.

79. Ljungberg M, Pretorius PH. SPECT/CT: an update on technological developments and clinical applications. Br J Radiol 2018;91:20160402.

80. Van Audenhaege K, Vandenberghe S, Deprez K, Vandeghinste B, Van Holen R. Design and simulation of a full-ring multi-lofthole collimator for brain SPECT. Phys Med Biol 2013;58:6317.

81. Buck A, Decristoforo C. Highlights lecture EANM 2015: the search for nuclear medicine's superheroes. Eur J Nucl Med Mol Imaging 2016;43:1910-27.

82. Chen Y, Vastenhouw B, Wu C, Goorden MC, Beekman FJ. Optimized image acquisition for dopamine transporter imaging with ultra-high resolution clinical pinhole SPECT. Phys Med Biol 2018;63:225002.

83. Branderhorst $W$, Vastenhouw B, van der Have F, Blezer E, Bleeker W, Beekman F. Targeted multi-pinhole SPECT. Eur J Nucl Med Mol Imaging 2011;38:552-61.

84. Bae J, Bae S, Jung YJ, Lee K, Kim Y, Joung J, Kim KM, Kim HJ. Experimental evaluation of a multi-pinhole collimator for a small organ by using a small-field-of-view 
gamma camera. J Korean Phys Soc 2017;70:416-23.

85. Bowsher J, Yan S, Roper J, Giles W, Yin FF. Onboard functional and molecular imaging: A design investigation for robotic multipinhole SPECT. Med Phys 2014;41:010701.

86. Wang B, van Roosmalen J, Kreuger R, Huizenga J, Beekman FJ, Goorden MC. Characterization of a multipinhole molecular breast tomosynthesis scanner. Phys Med Biol. 2020. doi:10.1088/1361-6560/ab9eff.

87. Uzun Ozsahin D, Blackberg L, Moghadam N, El Fakhri $\mathrm{G}$, Sabet H. GATE simulation of a high-performance stationary SPECT system for cardiac imaging. Lyon, France: IEEE Nuclear Science Symposium, Medical Imaging Conference Record, 2016.

88. Sabet H, Backberg L, Uzun Ozsahin D, El Fakhri G. Novel laser processed CsI:Tl detector for SPECT. Med Phys 2016;43:2630.

89. Erlandsson K, Kacperski K, van Gramberg D, Hutton BF. Performance evaluation of D-SPECT: A novel SPECT system for nuclear cardiology. Phys Med Biol 2009;54:2635-49.

90. Jan S, Santin G, Strul D, Staelens S, Assie K, Autret D, et al. GATE: a simulation toolkit for PET and SPECT. Phys Med Biol 2004;49:4543-61.

91. Zeraatkar N, Kalluri KS, Konik A, Mukherjee JM, Dey J, Goding JC, He Y, Fromme TJ, Auer B, Banerjee S, Mok G, Zubal GI, Furenlid LR, King MA. Preliminary Investigation of Axial and Angular Sampling in MultiPinhole AdaptiSPECT-C with XCAT Phantoms. Lyon, France: IEEE NSS/MIC Conf. Record ,2017.

92. Momsen NC, Richards G, King MA, Zeraatkar N, Furenlid LR. Design of a selectable pinhole module for use in adaptive SPECT collimators. Lyon, France: IEEE Nuclear Science Symposium and Medical Imaging Conference Record, 2018.

93. Zeraatkar N, Kalluri K, Furenlid LR, Zubal GI, King MA. Temporal shuttering of apertures to enhance axial and

Cite this article as: Ozsahin I, Chen L, Könik A, King MA, Beekman FJ, Mok GSP. The clinical utilities of multi-pinhole single photon emission computed tomography. Quant Imaging Med Surg 2020;10(10):2006-2029. doi: 10.21037/qims-19-1036 angular sampling in a multi-pinhole SPECT system for brain imaging. J Nucl Med 2018;59:abst 585.

94. Ogawa K, Ichimura Y. Simulation study on a stationary data acquisition SPECT system with multi-pinhole collimators attached to a triple-head gamma camera system. Ann Nucl Med 2014;28:716-24.

95. Bhusal N, Dey J, Xu J, Kalluri K, Konik A, Mukherjee JM, Pretorius PH. Performance analysis of a high-sensitivity multi-pinhole cardiac SPECT system with hemi-ellipsoid detectors. Med Phys 2019;46:116-26.

96. Dey J. Improvement of performance of cardiac SPECT camera using curved detectors with pinholes. IEEE Trans Nucl Sci 2012;59:334-7.

97. Kalluri K, Bhusal N, Shumilov D, Konik A, Mukherjee JM, Pretorius PH, Dey J. Multi-pinhole cardiac SPECT performance with hemi-ellipsoid detectors for two geometries. In: Proceedings of IEEE NSS-MIC, 2015.

98. Salvado D, Erlandsson K, Bousse A, Occhipinti M, Busca P, Fiorini C, Hutton B. Collimator design for a brain SPECT/ MRI insert. IEEE Trans Nucl Sci 2015;62:1716-24.

99. Busca P, Fiorini C, Butt AD, Occhipinti M, Peloso R, Quagliaet R al. Simulation of the expected performance of INSERT: A new multi-modality SPECT/MRI system for preclinical and clinical imaging. Nucl Instrum Methods Phys Res 2014;734:141-6.

100. Van Audenhaege K, Van Holen R, Vanhove C, Vandenberghe S. Collimator design for a multipinhole brain SPECT insert for MRI. Med Phys 2015;42:667989.

101. Bajaj N, Hauser RA, Grachev ID. Clinical utility of dopamine transporter single photon emission CT (DaT-SPECT) with (123I) ioflupane in diagnosis of parkinsonian syndromes. J Neurol Neurosurg Psychiatry 2013;84:1288-95.

102. Ono M, Watanabe H, Kitada A, Matsumura K, Ihara M, Saji H. Highly selective tau-SPECT imaging probes for detection of neurofibrillary tangles in Alzheimer's disease. Sci Rep 2016;6:34197. 